\title{
AN MILP MODEL FOR SAFE MULTI-FLOOR PROCESS PLANT LAYOUT USING THE DOMINO HAZARD INDEX
}

\author{
Jude O. Ejeh ${ }^{\mathrm{a}, \mathrm{b}}$, Songsong Liu ${ }^{\mathrm{c}}$, Lazaros G. Papageorgiou ${ }^{\mathrm{a}, *}$ \\ ${ }^{a}$ Centre for Process Systems Engineering, Department of Chemical Engineering, University \\ College London, Torrington place, London WC1E 7JE, United Kingdom \\ ${ }^{b}$ Department of Chemical \& Biological Engineering, The University of Sheffield, Mappin \\ Street, Sheffield, S1 3JD, United Kingdom \\ ${ }^{c}$ School of Management, Harbin Institute of Technology, Harbin 150001, China
}

\begin{abstract}
In this paper, an optimisation-based approach to obtain safe multi-floor process plant layout designs using the Domino Hazard Index (a sub-index of the Integrated Inherent Safety Index) is presented. A mixed integer linear programming (MILP) model is proposed to obtain the economically optimal multi-floor layout design considering connection by pipes, horizontal and vertical pumping of process fluids, purchase of land, fixed and area-dependent construction of floors, the financial risk associated with hazardous events and their escalation potential, and the installation of passive protection devices. Hazardous events such as pool fires, jet fires, flash fires, fireballs and blast waves resulting from explosions are considered using a novel and more realistic estimation of safety distances between equipment items. A bi-objective optimisation problem is also considered, minimising the layout costs and the total domino hazard index values for the plant, adopting the $\epsilon$-constraint method. The proposed model is then applied to an 11-unit case study susceptible to each of these hazardous events, obtaining results with the optimal layout and protection device configurations in a relatively short amount of time.
\end{abstract}

Keywords: multi-floor process plant layout, mixed integer linear programming (MILP), multi-objective optimisation, domino hazard index, safety

\section{Introduction}

The layout of a chemical process plant is important in defining the overall level of safety within the plant and its environment (Tugnoli et al., 2008). Safety is an issue of vital importance not at just at the stage of layout design but at

\footnotetext{
*Corresponding author. Tel: +44 (0)20 76792563

Email address: 1.papageorgiou@ucl.ac.uk (Lazaros G. Papageorgiou)
} 
every stage of chemical process design (Khan and Amyotte, 2004). Improper safety considerations can disrupt production activities, cause, injuries, fatalities, and also impact the plant environment and neighbouring areas negatively. However, up to $79 \%$ of process plant accidents have been attributed to design errors, the most critical being poor layout configurations (Kidam and Hurme, 2012).

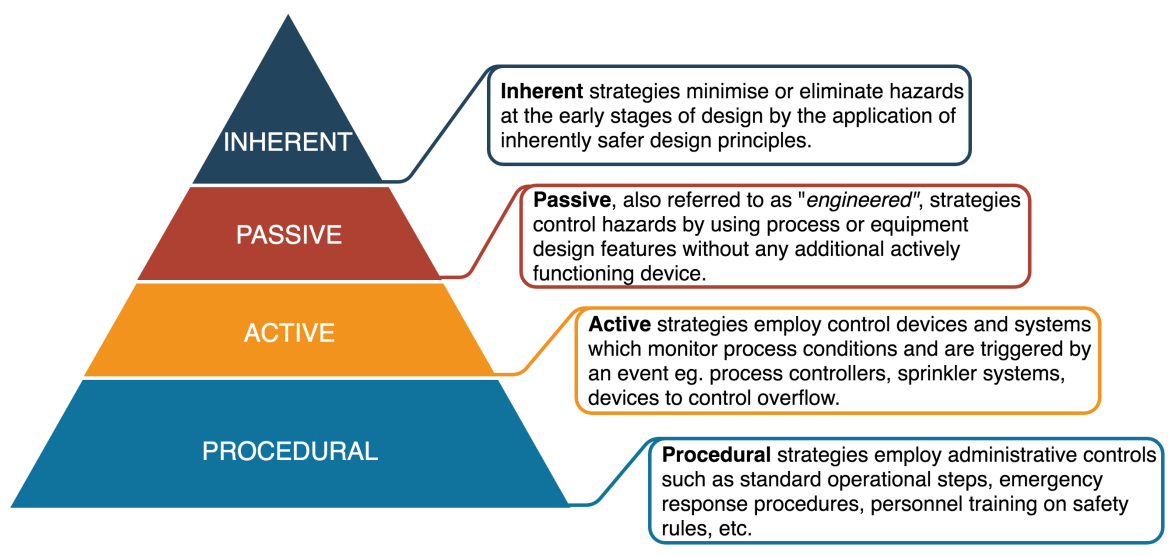

Figure 1: Process safety strategies

The current strategies in managing process safety and risk can be grouped into four categories (Jafari et al., 2018, Khan and Amyotte, 2004) as shown in Figure 1: inherent, passive (engineered), active and procedural. Inherently safer designs, adopted by the inherent strategy, are regarded as being at the top of the hierarchy in process safety management and one of the main future directions for loss prevention (Jafari et al., 2018). This is so as it has been identified that hazard prevention is much more significant when compared to reactions after accidents (Latifi et al., 2017). The principles adopted include (but are not limited to) the following (Jafari et al., 2018, Khan and Amyotte, 2004):

- minimization - reducing the quantity of hazardous materials;

- substitution - replacing materials with safer alternatives;

- attenuation - operating at safer conditions/bounds; and

- simplification - reducing the complexities in design and operation to prevent human errors.

When applied to layout designs, the principles/guidewords of effect are attenuation and simplification. This entails producing layout designs that provide safer conditions such as minimizing the domino potential of equipment items, and the consequences to neighbouring units, plant area and the environment; and that the layout of the plant is as simple as possible. An interpretation of simplicity 
can be related to the logical flow of process streams (Tugnoli et al., 2008), amongst others.

From an optimisation point of view, safety factors in layout designs have been considered in the past. A great deal of research have focused on the economic aspects - piping, construction and operational costs - associated with a layout configuration in single and multi-floor scenarios (Barbosa-Póvoa et al., 2002, Ejeh et al., 2018a;b; 2019b, Georgiadis and Macchietto, 1997, Guirardello and Swaney, 2005, Hwang and Lee, 2014, Papageorgiou and Rotstein, 1998, Park et al., 2011; 2018, Patsiatzis and Papageorgiou, 2002; 2003). However, an ideal plant layout design ought to establish a balance between risks and cost savings. Penteado and Ciric (1996) considered the financial risk associated with accidents as well as the installation of passive protection devices in an MINLP model. de Lira-Flores et al. (2019) proposed an MINLP model to simultaneously obtain the equipment layout, facility layout and safety instrumented system design with minimal risk to a process plant. Other authors used the quantitative risk assessment (QRA) methodology via a multi-stage approach: the first set of stages to quantify the risk of fires and explosions to equipment, its frequency and probability, and the ideal safety distances; and a final stage to obtain the optimal layout configuration (Ahumada et al., 2018, de Lira-Flores et al., 2018, Jung, 2016, Medina-Herrera et al., 2014). Díaz-Ovalle et al. (2013) also proposed a model using probit functions for risk estimation. The proposed model was able to determine the optimal layout and selection of mitigation system type for facilities prone to toxic release. Human risk considerations have also been included (Han et al., 2013), as well as employing the Dow's Fire and Explosion Index (F\&EI) system (Ejeh et al., 2019a, American Institute of Chemical Engineers, 1994, Park et al., 2018, Patsiatzis et al., 2004, Wang et al., 2017).

The Dow's F\&EI is a widely applied safety index in the chemical process industry. Using information on the chemical properties of materials within an equipment item, it estimates the associated hazards and potential economic risk such item poses to itself and other neighbouring items with or without protection devices installed (American Institute of Chemical Engineers, 1994). However, it does not account for other hazardous events apart from fires and explosions, and has been shown not to correlate well with known chemical plant disasters. In the review of safety indices applicable to chemical process design presented by Roy et al. (2016), the Domino Hazard Index (DHI) was also identified as a suitable index in layout decision making for chemical process plants.

The DHI gives a domino hazard ranking of a process plant equipment item on the basis of a set of scenarios, the distance between other equipment items, and physical and chemical properties. It is a sub-index of the Integrated Inherent Safety Index (I2SI) (Khan and Amyotte, 2005) which accounts for the hazard potential, inherent safety potential and add-on control requirements of a process. The DHI considers both inherent and passive measures and their effect on the domino escalation potential. Fire, explosion, toxic release and other closely related scenarios are considered. de Lira-Flores et al. (2014) presented a mixed integer non-linear programming (MINLP) approach for single floor layout designs 
based on this index for pool fires, jet fires, flash fires, fireballs and blast waves events. The resulting model was solved using a local MINLP solver and globally optimally results were not guaranteed. Latifi et al. (2017) also proposed a similar MINLP model using a Bat meta-heuristic algorithm to obtain solutions, and Wang et al. (2019) proposed a model with a sole objective to minimise the total DHI value of a plant.

The model proposed in this work seeks to build upon the work of de LiraFlores et al. (2014), presenting a more efficient mixed integer linear programming (MILP) model for safe layout configuration using the DHI. de Lira-Flores et al. (2014) considered connections by pipes, installation of protection device and the domino escalation costs owing to pool fires, jet fires, flash fires, fireballs and blast wave events for single floor layout configurations. In this work, additional features including multi-floor layout configurations, tall-equipment items, vertical and horizontal pumping costs, and a more realistic estimation of separation distances for the DHI metrics are considered. Multi-floor configurations are a growing consideration owing to increasing land costs, problems of availability of land, and the fact that a number of chemical process plant structures, by default, have multiple floors e.g. offshore plants (Hosseini-Nasab et al., 2018). Pumping costs are important as they are a key contributor to plant operating costs from a layout perspective, especially vertical pumping costs prevalent in multi-floor layouts. Chemical process plants are also commonly known to have tall equipment items e.g. distillation columns, flare stacks, furnace, that do not fit to single floors but extend well above the standard floor height through several floors. It becomes important, not only to properly describe these equipment types, but also more accurately estimate the separation distance between these units for determining the level of risk and/or the need for protection devices.

In the next section more detail is given to the problem being considered; in section 3 the steps for calculating the DHI are explained; the proposed model is described in section 4; and a case study is presented in section 5 to show the proposed model's performance, its new features, how it compares to previous models as well as a bi-objective consideration. Finally, concluding remarks on major findings are highlighted in section 6 .

\section{Problem Description}

This work seeks to obtain the multi-floor layout configuration of a chemical process plant with safety considerations. The latter is quantified using the DHI. This safety index assesses the effects hazards from process plant equipment items have on their domino escalation potential, with respect to their separation distances from neighbouring items and the presence of passive protection devices. The proposed mathematical model assumes that:

- each equipment item has a rectangular geometry;

- each item is connected to others from its geometrical centre in the $x-y$ plane, and from a predefined height along the z-plane, based on design specifications; 
- safety distances between items are measured from their boundaries and taken as the Tchebychev distance in all $\mathrm{x}, \mathrm{y}$ and $\mathrm{z}$ planes;

- equipment items are allowed to rotate $90^{\circ}$ angles about the $\mathrm{x}$-y plane as deemed optimal but must start at the base of the floor they are assigned;

- floors are numbered bottom-up with a fixed floor height;

- equipment items that exceed the fixed floor height are allowed to extend through successive floors;

- separating floor structures can act as passive protection devices against certain hazards.

The problem is described as follows:

\section{Given:}

- a set of process units, their type, dimensions, damage index values and process conditions;

- a set of likely primary events for each equipment item $i$;

- a set of potential floors;

- connectivity network amongst process units;

- cost data (equipment, connection, pumping, land, construction, protection devices);

- space and unit allocation limitations;

to determine:

- total number of floors required;

- base land area;

- area of floors;

- plot layout;

- type and number of protective devices required;

- DHI value for each equipment;

so as to: minimise the total layout and safety costs.

\section{Domino Hazard Index}

The Domino Hazard Index is estimated as shown in Figure 2. A description of each step and its mathematical formulation are described below. 


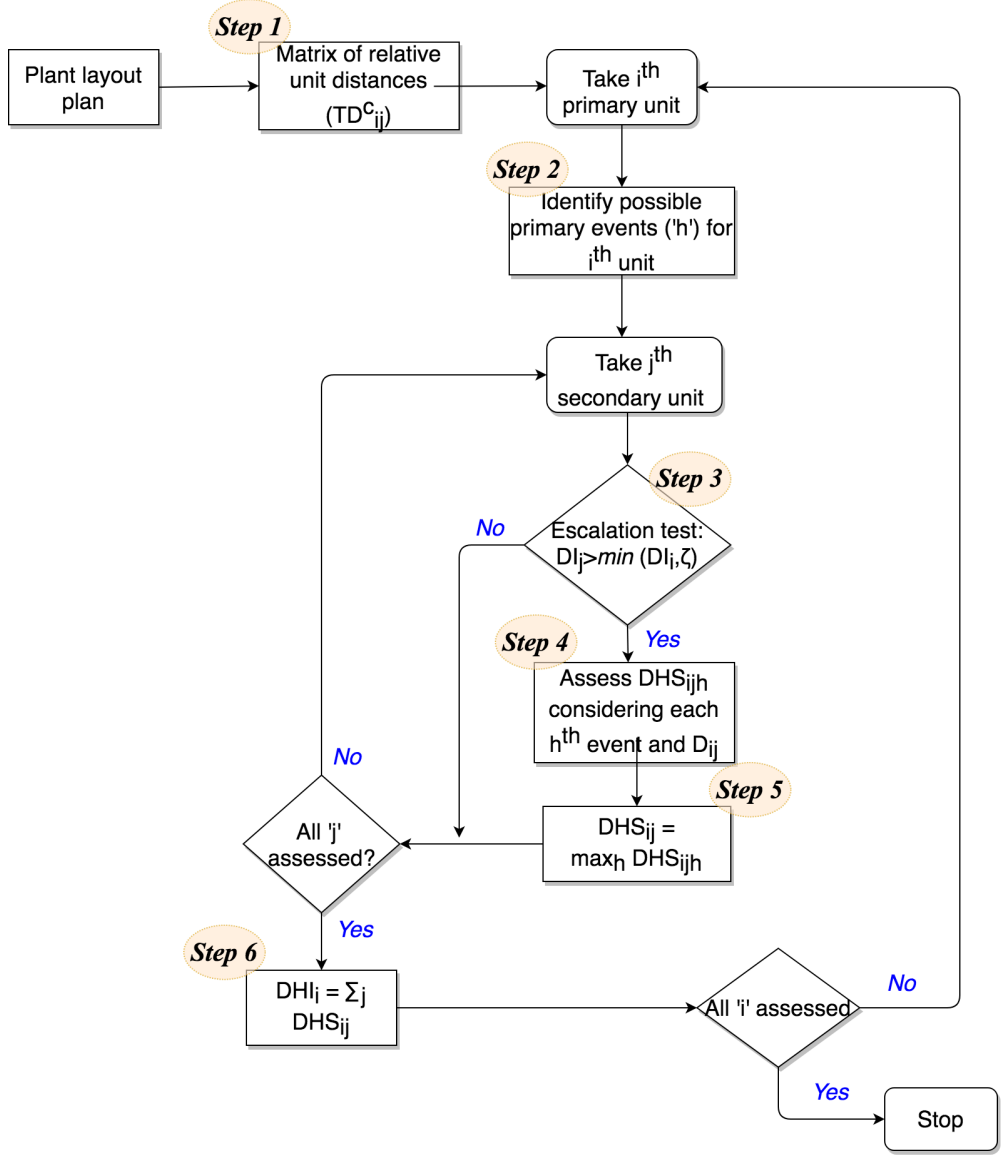

Figure 2: Flow diagram of DHI assessment

\subsection{Step 1}

The separation distance $\left(T D_{i j}^{s}\right)$ between equipment item pairs should be evaluated.

\subsection{Step 2}

For each unit $i$, all possible primary events $h$ that can trigger domino effects should be identified.

These primary events, as outlined by Cozzani et al. (2006), are listed in Table 1. Based on the nature/type of the equipment and/or material it processes, a set of primary event can be identified for each unit. 
Table 1: Escalation vectors and expected secondary scenarios for different primary events

\begin{tabular}{|c|c|c|}
\hline Primary events & Escalation vector & Expected secondary scenarios \\
\hline Pool fire & Radiation, fire impingement & $\begin{array}{l}\text { Jet fire, pool fire, } \\
\text { BLEVE }^{1} \text {, toxic release }\end{array}$ \\
\hline Jet fire & Radiation, fire impingement & $\begin{array}{l}\text { Jet fire, pool fire, } \\
\text { BLEVE }^{1} \text {, toxic release }\end{array}$ \\
\hline Fireball & Radiation, fire impingement & Tank fire \\
\hline Flash fire & Fire impingement & Tank fire \\
\hline Blast wave & overpressure & $\mathrm{All}^{2}$ \\
\hline
\end{tabular}

${ }^{1}$ BLEVE - boiling liquid expanding vapour explosion

${ }^{2}$ All - any of the events listed under the 'Primary events' column

\subsection{Step 3}

For each secondary unit $j$, the values of the Damage Index (DI) for both units primary unit $i$, and $j$ should be compared in order to identify units having significant potential to increase adverse scenarios.

The Damage Index is calculated as described by Khan and Amyotte (2004). The secondary units are selected based on the following condition:

$$
\Gamma^{H}=\left\{(i, j): D I_{j}>\min \left(D I_{i}, \zeta\right)\right\}
$$

where the value of $\zeta$ defines the lower limit of $D I$ for units considered as highly hazardous. The set $\Gamma^{H}$ defines the pairs of equipment items $(i, j)$ where $i$ is the primary item and hazardous event source, and $j$ is the secondary item susceptible to the domino effects from the event on $i$.

\subsection{Step 4}

The Domino Hazard Score $\left(D H S_{i j e}^{e}\right)$ for each identified event $e$ (Step 2) from unit $i$ to unit $j$ should be evaluated.

The Domino Hazard Score $\left(D H S_{i j e}^{e}\right)$ is assigned a value of 10 for a highly probably escalation of event $e$ and 0 for the inherently "safest" level for domino escalation. This score is evaluated for every identified primary event's escalation vector. A list of rules for events listed in Table 1 are outlined below. These rules help to estimate the values of $D H S_{i j e}^{e}$ for both inherent and passive measures.

\subsubsection{Flash fire $(F F)$}

For FF events, the score $D H S_{i j e}^{e}$ is 0 or 10 depending on whether the secondary unit $j$ is within direct reach of the flame envelope $\left(d_{e i}^{F}\right)$. No passive protection devices are considered, and assuming the flame originates from the outer surface of unit $i$ :

$$
D H S_{i j e}^{e}=\left\{\begin{array}{ll}
0, & T D_{i j}^{s}>d_{e i}^{F} \\
10, & T D_{i j}^{s} \leq d_{e i}^{F}
\end{array} \quad \forall(i, j) \in \Gamma^{H}, j \in I^{V}, e \in\{F F\}\right.
$$

where $I^{V}$ is the set of units likely to release flammable vapours. 


\subsubsection{Fire ball (FB)}

For fire ball events, fire impingement is likely to affect only atmospheric units $\left(i \in I^{A}\right)$ (de Lira-Flores et al., 2014) within the FB radius $d_{e i}^{F}$. The presence of passive devices such as fire insulation (FI) and fire resistant walls (FW) reduce the hazard score:

$$
D H S_{i j e}^{e}=\left\{\begin{array}{ll}
0, & T D_{i j}^{s}>d_{e i}^{F} \\
5, & T D_{i j}^{s} \leq d_{e i}^{F} \text { FI installed } \\
10, & T D_{i j}^{s} \leq d_{e i}^{F} \text { FI not installed }
\end{array} \quad \forall(i, j) \in \Gamma^{H}, j \in I^{A}, e \in\{F B\}\right.
$$

\subsubsection{Pool fire (PF) and Jet fire (JF)}

From Table 1, escalations vectors due to pool and jet fires are by fire impingement and heat radiation. With fire impingement, no passive protection device is considered adequate to reduce the DHS for any unit within the flame envelope $\left(d_{e i}^{F}\right)$, as small defects can nullify the protective behaviour (Tugnoli et al., 2008). For escalation events due to heat radiation, the DHS is influenced by the secondary unit's distance from the flame envelope $d r_{i j e}$, the scenario (jet or pool fire) and the characteristics of the unit (atmospheric or pressurised equipment) (Figure 3). Tugnoli et al. (2008) showed the DHS to be non-linear and inversely varied with the separation distance for atmospheric and pressurized units in JF and PF scenarios. These variations can be captured using a piece-wise linear approximation (de Lira-Flores et al., 2014).

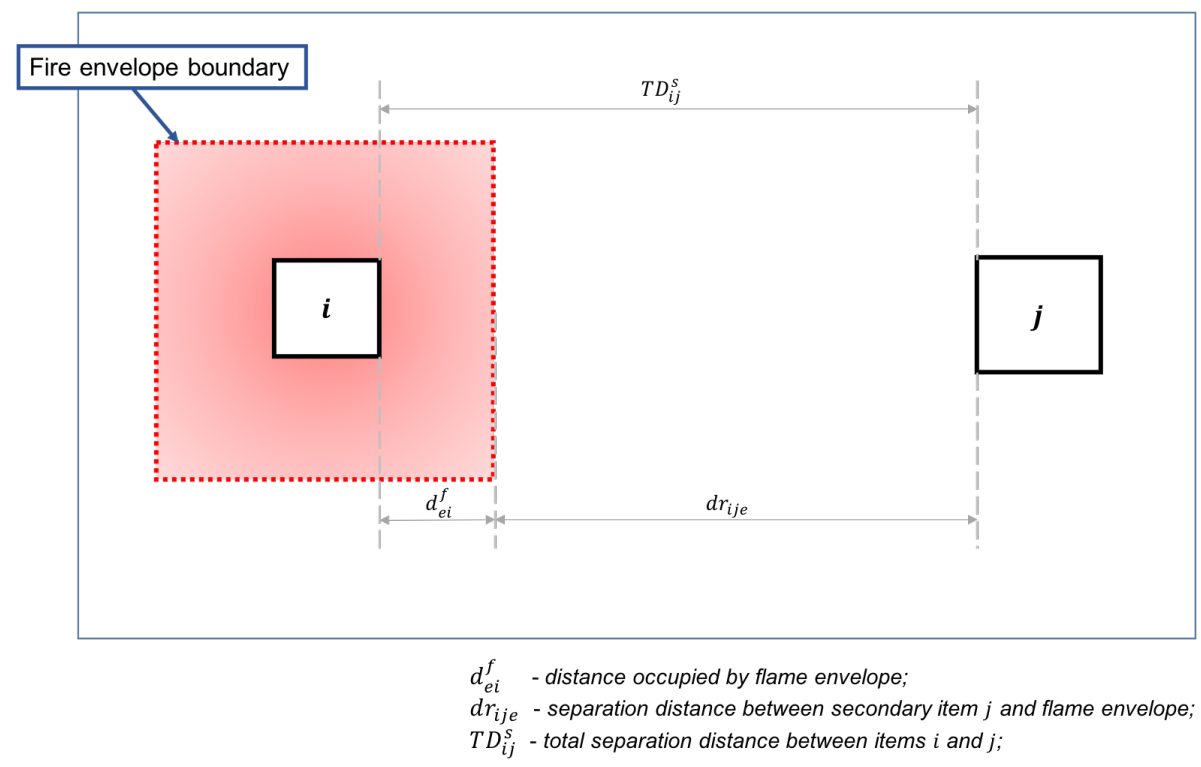

Figure 3: Distance calculations for Pool/Jet fire events 
For atmospheric equipment items:

$$
D H S_{i j e}^{e}=\left\{\begin{array}{ll}
0, & d r_{i j e}>d_{e j}^{S} \\
1, & d r_{i j e} \leq d_{e j}^{S} \mathrm{FW} \text { installed } \\
0<d r_{i j e} \leq d_{e j}^{S} \\
10-\sum_{\hat{q}=1}^{4} \kappa_{i h e \hat{q}}^{A} \cdot \mu_{i j h e \hat{q}}, \\
\& d r_{i j e}=\sum_{q=1}^{4} \lambda_{i h e \hat{q}}^{A} \cdot \mu_{i j h e \hat{q}} \\
10, & d r_{i j e} \leq 0
\end{array} \quad \forall(i, j) \in \Gamma^{H}\right.
$$

and for pressured equipment items:

$$
D H S_{i j e}^{e}=\left\{\begin{array}{ll}
0, & d r_{i j e}>d_{e j}^{S} \\
1, & d r_{i j e} \leq d_{e j}^{S} \mathrm{FW} \text { installed } \\
0<d r_{i j e} \leq d_{e j}^{S} & \& d r_{i j e}=\sum_{q=1}^{4} \lambda_{i h e \hat{q}}^{P} \cdot \mu_{i j h e \hat{q}} \\
10-\sum_{\hat{q}=1}^{4} \kappa_{i h e \hat{q}}^{P} \cdot \mu_{i j h e \hat{q}}, & d r_{i j e} \leq 0
\end{array} \quad \forall(i, j) \in \Gamma^{H}\right.
$$

where the values of the parameters $\kappa_{i h e \hat{q}}^{A}, \lambda_{i h e \hat{q}}^{A}$ and $\kappa_{i h e \hat{q}}^{P}, \lambda_{i h e \hat{q}}^{P}$ are shown in Table B.1 (Appendix ) for pressurised and atmospheric equipment respectively for both unprotected (HZ2) and fire-insulated scenarios; $d_{e i}^{S}$ is the safety distance given by Cozzani et al. (2006) for pool fires (15m - pressurised equipment, $50 \mathrm{~m}$ - atmospheric equipment) and jet fires (25m - pressurised equipment, $50 \mathrm{~m}$ atmospheric equipment); and $\mu_{i j h e \hat{q}}$ are variables satisfying:

- $\mu_{i j h e, 1} \leq 1$,

- $\mu_{i j h e, 4} \geq 0$ and

- $\mu_{i j h e, \hat{q}+1} \leq B_{i j h e \hat{q}}^{F} \leq \mu_{i j h e \hat{q}}$.

The distance from the secondary unit to the flame source is given as:

$$
d r_{i j e}=T D_{i j}^{S}-d_{e i}^{F} \quad \forall(i, j) \in \Gamma^{H}, e \in\{P F, J F\}
$$

\subsubsection{Blast wave $(B W)$}

Blast wave events encompass all primary events that result in an explosion - mechanical explosion, confined explosion, boiling liquid expanding vapour explosion (BLEVE), vapour cloud explosion (VCE) - with overpressure being the resulting escalation vector. Overpressure is the pressure, over and above the atmospheric pressure, caused by a shock wave from an explosion. The Domino Hazard Score for blast wave $\left(D H S_{i j e}^{e}\right)$ is calculated as a function of the static 
peak overpressure, and is a function of the distance from the explosion source $\left(d r_{i j e}\right)$ :

$$
D H S_{i j e}^{e}=\left\{\begin{array}{ll}
0, & d r_{i j e}>u_{i}^{B W} \\
1, & d r_{i j e} \leq u_{i}^{B W} \text { BWl installed } \\
\alpha_{i}^{B W} d r_{i j e}+\beta_{i}^{B W} & l_{i}^{B W} \leq d r_{i j e} \leq u_{i}^{B W} \\
10, & d r_{i j e}<l_{i}^{B W}
\end{array} \forall(i, j) \in \Gamma^{H}, e \in\{B W\}\right.
$$

where:

$$
\begin{aligned}
\alpha_{i}^{B W} & =\frac{10}{l_{i}^{B W}-u_{i}^{B W}} \\
\beta_{i}^{B W} & =-\alpha_{i}^{B W} u_{i}^{B W}
\end{aligned}
$$

The distance between equipment items is given by:

$$
d r_{i j e}=T D_{i j}^{S} \quad \forall(i, j) \in \Gamma^{H}, e \in\{B W\}
$$

as it is assumed that the explosion source is at the surface of the primary equipment item.

For pressurized items $j$, DHS is calculated as follows:

$$
D H S_{i j e}^{e}=\left\{\begin{array}{ll}
0, & d r_{i j e}>u_{i}^{B W} \\
1, & d r_{i j e} \leq u_{i}^{B W} \\
10, & d r_{i j e} \leq u_{i}^{B W}
\end{array} \text { BWl installed } \forall(i, j) \in \Gamma^{H}, e \in\{B W\}\right.
$$

\subsection{Step 5}

The Domino Hazard Score for each unit pair $(i, j)$ should then be evaluated. This value is taken as the highest value amongst all evaluated hazardous events:

$$
D H S_{i j} \geq D H S_{i j e}^{e} \quad \forall(i, j) \in \Gamma^{H}
$$

\subsection{Step 6}

As a final step, the Domino Hazard Index for each unit $i$ should then be calculated.

This is evaluated as follows:

$$
D H I_{i}=\sum_{j:(i, j) \in \Gamma^{H}} D H S_{i j} \quad \forall i
$$

To incorporate these steps into an optimisation model, additional constraints are included to describe the costs associated with passive measures to reduce domino effects, as well as the damage costs owing to the domino escalation of an event. 


\section{Mathematical Formulation}

In this section, the proposed mathematical model is presented in detail. The proposed model is an extension of the MILP model in Ejeh et al. (2019b) - model OPTL - which determines the optimal multi-floor process plant layout configuration for a set of process plant equipment items with considerations for piping, horizontal and vertical pumping, area-dependent land purchase, and fixed and area-dependent floor construction costs. Additional constraints are included to evaluate equipment separation distances, aside from connection distances, for safety metric calculations, hazard scenarios as outlined in Step 4 (section 3), and the purchase and installation of protection devices.

\subsection{Floor constraints}

In addition to the floor constraints by Ejeh et al. (2019b) outlined in the appendix, the following are introduced.

To ensure that the value of the binary variable $N_{i j}$ is 1 only when two items are assigned to the same floor, equation (A.4) is replaced by (14) - (20). For equipment items that occupy one floor:

$$
\begin{array}{ll}
N_{i j} \geq V_{i k}+V_{j k}-1 & \forall i \notin I^{T}, j \notin I^{T}, j>i, k \\
N_{i j} \leq 1-V_{i k}+V_{j k} & \forall i \notin I^{T}, j \notin I^{T}, j>i, k \\
N_{i j} \leq 1+V_{i k}-V_{j k} & \forall i \notin I^{T}, j \notin I^{T}, j>i, k
\end{array}
$$

As tall equipment items are assigned to more than one floor, the above constraints will be infeasible if either $i$ or $j$ were tall equipment. A binary variable, $N_{i j k}^{\prime}$, is thus introduced for a floor by floor consideration to determine if two items are assigned to a common floor:

$$
\begin{array}{cc}
N_{i j k}^{\prime} \geq V_{i k}+V_{j k}-1 & \forall\left(i \in I^{T} \text { or } j \in I^{T}\right), j>i, k \\
N_{i j k}^{\prime} \leq \frac{V_{i k}+V_{j k}}{2} & \forall\left(i \in I^{T} \text { or } j \in I^{T}\right), j>i, k
\end{array}
$$

The variable $N_{i j}$ is then determined for item pairs with tall equipment items as:

$$
\begin{aligned}
& N_{i j k}^{\prime} \leq N_{i j} \quad \forall\left(i \in I^{T} \text { or } j \in I^{T}\right), j>i, k \\
& N_{i j} \leq \sum_{k} N_{i j k}^{\prime} \quad \forall\left(i \in I^{T} \text { or } j \in I^{T}\right), j>i
\end{aligned}
$$

Equation (21) is also written to account for all possible equipment item pairs:

$$
N_{i j}=N_{j i} \quad \forall i, j>i
$$

Equation (22) is included to guarantee that each existing floor has at least one equipment item assigned to it:

$$
W_{k} \leq \frac{\left(\sum_{i} S_{i k}^{S}\right)-1}{|I|}+1 \quad \forall k
$$




\subsection{Distance constraints}

The distance between equipment items are classified into two: connection distances, which are used to estimate pipe lengths and costs, and safety distances, used in evaluating the DHI, associated escalation costs and the set of protection devices to be installed.

\subsubsection{Connection distance constraints}

Connection distances are calculated as the rectilinear distances between equipment items as described by equations (A.17) - (A.19) in the appendix.

\subsubsection{Safety distance constraints}

The safety distance on the other hand is evaluated as the separation distance between equipment item boundaries and is obtained as follows.

Equations (A.17) and (A.18) are first re-written for highly hazardous pairs of equipment items $\left((i, j) \in \Gamma^{H}\right)$ :

$$
\begin{array}{ll}
R_{i j}-L_{i j}=x_{i}-x_{j} & \forall(i, j) \in \Gamma^{H} \\
A_{i j}-B_{i j}=y_{i}-y_{j} & \forall(i, j) \in \Gamma^{H}
\end{array}
$$

Binary variables $W_{i j}^{X}$ and $W_{i j}^{Y}$ force one of $R_{i j}$ or $L_{i j}$ (equation (23)), and $A_{i j}$ or $B_{i j}$ (equation (24)) to zero respectively by equations (25) - (28).

$$
\begin{aligned}
& R_{i j} \leq B M \cdot W_{i j}^{X} \quad \forall(i, j) \in \Gamma^{H} \\
& L_{i j} \leq B M \cdot\left(1-W_{i j}^{X}\right) \quad \forall(i, j) \in \Gamma^{H} \\
& A_{i j} \leq B M \cdot W_{i j}^{Y} \quad \forall(i, j) \in \Gamma^{H} \\
& B_{i j} \leq B M \cdot\left(1-W_{i j}^{Y}\right) \quad \forall(i, j) \in \Gamma^{H}
\end{aligned}
$$

For the vertical safety distance, the following conditions should be satisfied as shown in Figure 4:

- if two items $i$ and $j$ are on the same floor, the vertical distance is taken to be zero;

- if $i$ is on a higher floor than $j$, the vertical distance is taken from the top of $j$ to the bottom of $i$; and

- if $i$ is on a lower floor than $j$, the vertical distance is taken from the top of $i$ to the bottom of $j$. 


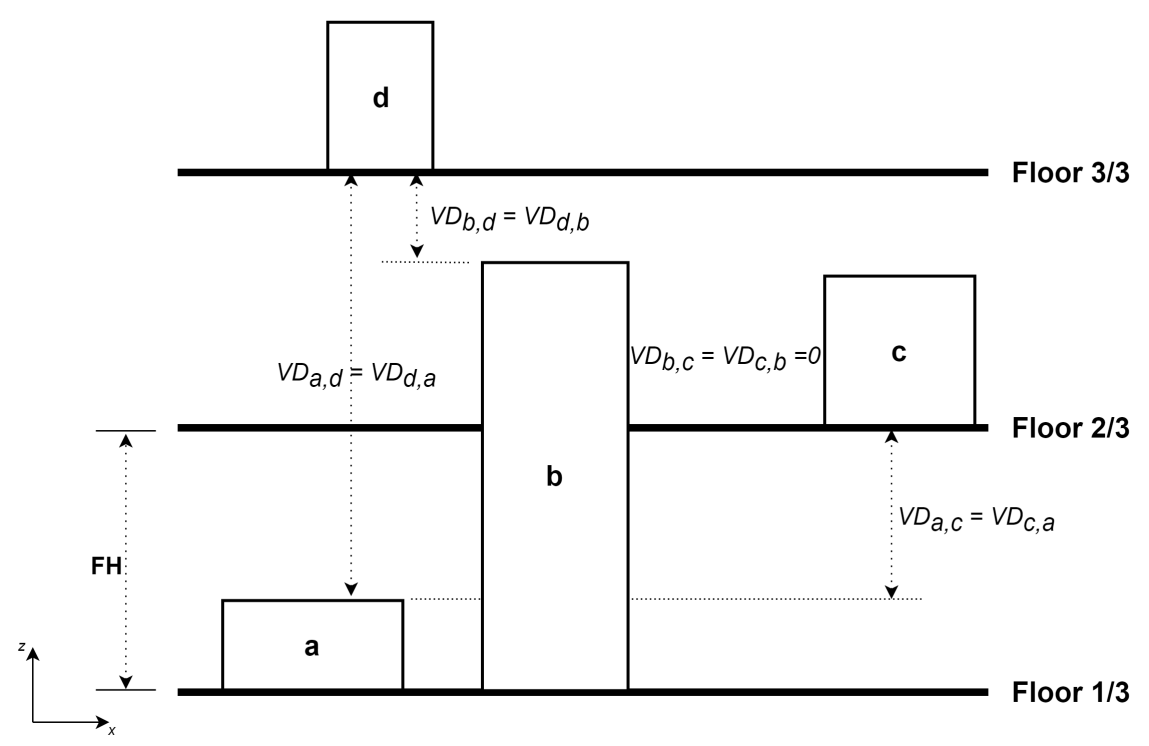

Figure 4: Vertical safety distance between equipment items

These conditions are represented mathematically by equation (29) for the vertical separation distance, $V D_{i j}$, between any two items $i$ and $j$.

$$
V D_{i j}\left\{\begin{array}{l}
\geq 0, \quad \text { when } N_{i j}=0 \\
=0, \quad \text { when } N_{i j}=1
\end{array}\right.
$$

For situations were items $i$ and $j$ are on different floors $\left(N_{i j}=0\right)$, the vertical distance is determined as follows. Equations (30) or (31) determine the separation distances between items $i$ and $j$ if $i$ is above or below $j$ respectively (Figure 4).

$$
\begin{array}{ll}
U p_{i j}=F H \sum_{k}(k-1)\left(S_{i k}^{S}-S_{j k}^{S}\right)-\gamma_{j} & \forall(i, j) \in \Gamma^{H}, N_{i j}=0 \\
D n_{i j}=F H \sum_{k}(k-1)\left(S_{j k}^{S}-S_{i k}^{S}\right)-\gamma_{i} & \forall(i, j) \in \Gamma^{H}, N_{i j}=0
\end{array}
$$

The vertical separation distance between the two items $i$ and $j$ will then be the maximum value of $U p_{i j}$ and $D n_{i j}$ :

$V D_{i j}=\max \left(U p_{i j}, D n_{i j}\right)=D n_{i j}+\max \left(U p_{i j}-D n_{i j}, 0\right) \quad \forall(i, j) \in \Gamma^{H}, N_{i j}=0$

As equations (30) - (32) cannot be implemented directly using conventional optimization software, linear reformulations are proposed. Equation (33) helps 
to determine $\max \left(U p_{i j}-D n_{i j}, 0\right)$ where $\eta_{i j}^{U}$ and $\eta_{i j}^{D}$ are positive variables:

$$
\begin{array}{rlrl}
\eta_{i j}^{U}-\eta_{i j}^{D} & =2 F H \sum_{k}(k-1)\left(S_{i k}^{S}-S_{j k}^{S}\right)+\gamma_{i}-\gamma_{j} & & \forall(i, j) \in \Gamma^{H} \\
\eta_{i j}^{U} \leq B M \cdot W_{i j}^{Z} & & \forall(i, j) \in \Gamma^{H} \\
\eta_{i j}^{D} & \leq B M \cdot\left(1-W_{i j}^{Z}\right) & & \forall(i, j) \in \Gamma^{H}
\end{array}
$$

Equations (34) and (35) ensure only one of $\eta_{i j}^{U}$ or $\eta_{i j}^{D}$ takes a non-zero value. The total vertical distance is then evaluated as:

$$
V D_{i j}=F H \sum_{k}(k-1)\left(S_{j k}^{S}-S_{i k}^{S}\right)-\gamma_{i}+\eta_{i j}^{U} \quad \forall(i, j) \in \Gamma^{H}
$$

To also account for equipment items on the same floor $\left(N_{i j}=1\right)$, equation (36) is rewritten as:

$$
\begin{array}{cc}
V D_{i j} \leq F H \sum_{k}(k-1)\left(S_{j k}^{S}-S_{i k}^{S}\right)-\gamma_{i}+\eta_{i j}^{U}+B M \cdot N_{i j} & \forall(i, j) \in \Gamma^{H} \\
V D_{i j} \geq F H \sum_{k}(k-1)\left(S_{j k}^{S}-S_{i k}^{S}\right)-\gamma_{i}+\eta_{i j}^{U}-B M \cdot N_{i j} & \forall(i, j) \in \Gamma^{H} \\
V D_{i j} \leq B M \cdot\left(1-N_{i j}\right) \quad \forall(i, j) \in \Gamma^{H} &
\end{array}
$$

Equations (37) - (39) ensure $V D_{i j}$ takes a non-zero value only if items $i$ and $j$ are located on different floors.

The horizontal safety distances are also estimated using a similar assumption - from the boundaries of each equipment item, as opposed to their geometrical centres (equations (A.17) and (A.18)). A value of zero is assigned to this distance if the boundaries of an item $i$ is not strictly to the right or left (on the x plane), or above or below (on the y plane) of $j$ (Figure 5). 


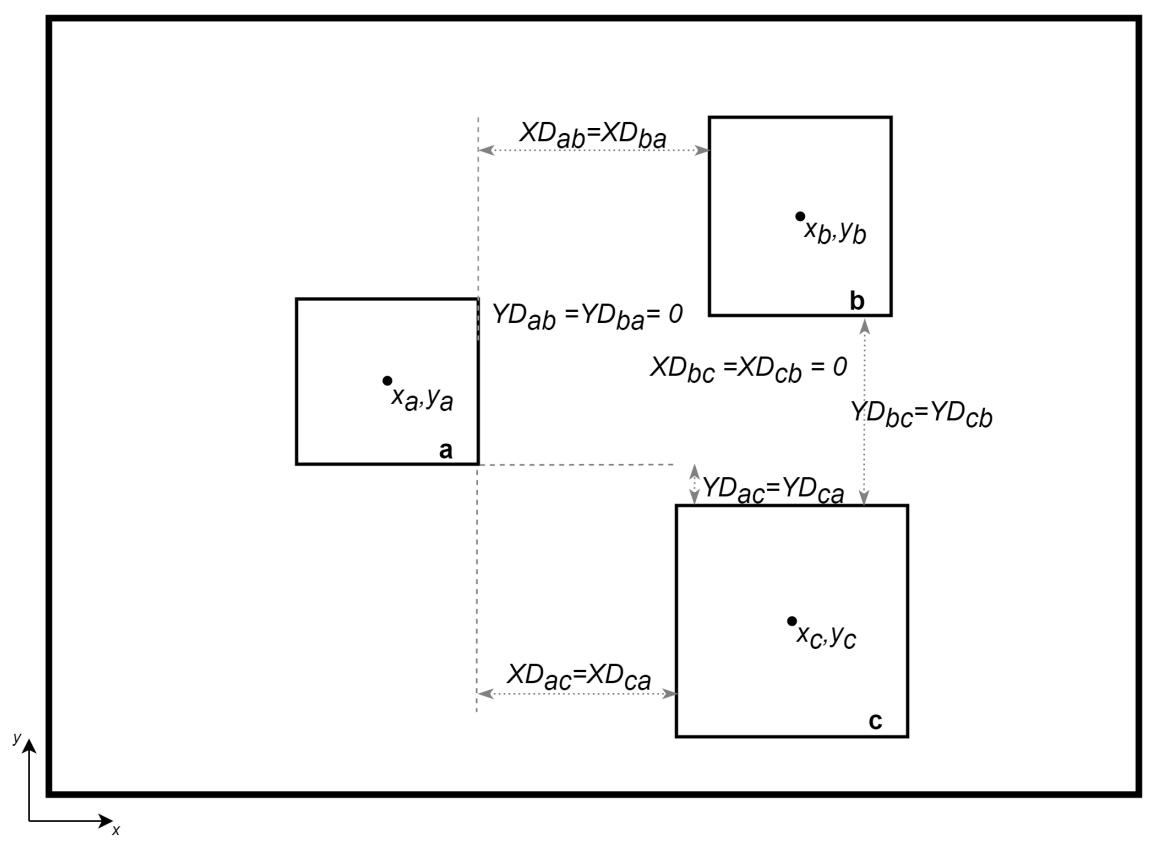

Figure 5: Horizontal safety distance between equipment items

$$
\begin{array}{rr}
y_{i}-y_{j}+2 B_{i j} \geq\left(\frac{d_{i}+d_{j}}{2}\right)-B M \cdot\left(1-W_{i j}^{Y O}\right) & \forall(i, j) \in \Gamma^{H} \\
Y D_{i j} \leq A_{i j}+B_{i j}-\left(\frac{d_{i}+d_{j}}{2}\right)+B M \cdot\left(1-W_{i j}^{Y O}\right) & \forall(i, j) \in \Gamma^{H} \\
Y D_{i j} \geq A_{i j}+B_{i j}-\left(\frac{d_{i}+d_{j}}{2}\right)-B M \cdot\left(1-W_{i j}^{Y O}\right) & \forall(i, j) \in \Gamma^{H} \\
Y D_{i j} \leq B M \cdot W_{i j}^{Y O} \quad \forall(i, j) \in \Gamma^{H} &
\end{array}
$$

Equation (40) ensures that if the opposing boundaries of item $i$ is strictly above or below item $j$ and not overlapping in any region on the y plane, $W_{i j}^{Y O}$ takes a value of 1 . Equations (41) - (43) ensure that the distance between the boundaries of items $i$ and $j\left(Y D_{i j}\right)$ on the y plane only takes a positive value if both items do not overlap along any region of the y plane, else, a value of zero 
is assigned. A similar set of constraints apply for the x plane ((44) - (47)).

$$
\begin{array}{rr}
x_{i}-x_{j}+2 L_{i j} \geq\left(\frac{l_{i}+l_{j}}{2}\right)-B M \cdot\left(1-W_{i j}^{X O}\right) & \forall(i, j) \in \Gamma^{H} \\
X D_{i j} \leq R_{i j}+L_{i j}-\left(\frac{l_{i}+l_{j}}{2}\right)+B M \cdot\left(1-W_{i j}^{X O}\right) & \forall(i, j) \in \Gamma^{H} \\
X D_{i j} \geq R_{i j}+L_{i j}-\left(\frac{l_{i}+l_{j}}{2}\right)-B M \cdot\left(1-W_{i j}^{X O}\right) & \forall(i, j) \in \Gamma^{H} \\
X D_{i j} \leq B M \cdot W_{i j}^{X O} \quad \forall(i, j) \in \Gamma^{H} &
\end{array}
$$

A binary variable $W_{i j}^{X O}$ is introduced having a value of 1 if the opposing boundaries of item $i$ is strictly to the right or left of $j$.

The total safety distances between equipment items $i$ and $j$ is then calculated as the Tchebychev distance between the equipment boundaries of such items in all $\mathrm{x}-, \mathrm{y}-$ and z-planes. This distance metric is selected as it is a closer estimate to the Euclidean distance between equipment item boundaries when compared to the rectilinear distance. The Euclidean distance estimation however introduces a non-linear term which makes the model difficult to solve. The total safety distance is thus calculated as:

$$
T D_{i j}^{s}=\max \left(X D_{i j}, Y D_{i j}, V D_{i j}\right) \quad \forall(i, j) \in \Gamma^{H}
$$

Equation (48) can also be expressed as $\max \left(\max \left(X D_{i j}, Y D_{i j}\right), V D_{i j}\right)$ which is linearised as follows.

The first part $X Y_{i j}^{\max }=\max \left(X D_{i j}, Y D_{i j}\right)$ is linearised using equations (49) (55):

$$
\begin{array}{cc}
X Y_{i j}^{\max } \leq X D_{i j}+B M \cdot\left(1-M B_{i j}^{X Y}\right) & \forall(i, j) \in \Gamma^{H} \\
X Y_{i j}^{\max } \geq X D_{i j}-B M \cdot\left(1-M B_{i j}^{X Y}\right) & \forall(i, j) \in \Gamma^{H} \\
X Y_{i j}^{\max } \leq Y D_{i j}+B M \cdot M B_{i j}^{X Y} & \forall(i, j) \in \Gamma^{H} \\
X Y_{i j}^{\max } \geq Y D_{i j}-B M \cdot M B_{i j}^{X Y} & \forall(i, j) \in \Gamma^{H} \\
X D_{i j} \geq Y D_{i j}-B M \cdot\left(1-M B_{i j}^{X Y}\right) & \forall(i, j) \in \Gamma^{H} \\
Y D_{i j} \geq X D_{i j}-B M \cdot M B_{i j}^{X Y} & \forall(i, j) \in \Gamma^{H} \\
X Y_{i j}^{\max } \leq B M \cdot\left(W_{i j}^{X O}+W_{i j}^{Y O}\right) & \forall(i, j) \in \Gamma^{H}
\end{array}
$$

where $M B_{i j}^{X Y}$ is a binary variable equal to 1 when $X D_{i j} \geq Y D_{i j}$. Integer cuts are included to select $Y D_{i j}$ if $X D_{i j}$ is zero (equations (56)), and $X D_{i j}$ if both $X D_{i j}$ and $Y D_{i j}$ are zero (equations (57)):

$$
\begin{gathered}
M B_{i j}^{X Y} \leq W_{i j}^{X O}+W_{i j}^{Y O} \quad \forall(i, j) \in \Gamma^{H} \\
W_{i j}^{X O} \geq M B_{i j}^{X Y} \quad \forall(i, j) \in \Gamma^{H}
\end{gathered}
$$


The second part of equation $(48), \max \left(X Y_{i j}^{\max }, V D_{i j}\right)$ is linearised as:

$$
\begin{gathered}
T D_{i j}^{s}=X Y_{i j}^{\max }+Z D_{i j}^{+} \quad \forall(i, j) \in \Gamma^{H} \\
Z D_{i j}^{+}-Z D_{i j}^{-}=V D_{i j}-X Y_{i j}^{\max } \quad \forall(i, j) \in \Gamma^{H} \\
Z D_{i j}^{+} \leq B M \cdot M B_{i j}^{Z} \quad \forall(i, j) \in \Gamma^{H} \\
Z D_{i j}^{-} \leq B M \cdot\left(1-M B_{i j}^{Z}\right) \quad \forall(i, j) \in \Gamma^{H} \\
M B_{i j}^{Z} \leq 1-N_{i j} \quad \forall(i, j) \in \Gamma^{H}
\end{gathered}
$$

where $M B_{i j}^{Z}$ is a binary variable indicating when $V D_{i j} \geq X Y_{i j}^{\max }$.

\subsection{Flash fire $(F F)$}

Constraints are introduced to represent each of the possible hazardous event described in section 3. For each event, a new variable $d_{i j h e}^{H}$ is introduced expressing the distance between $i$ and $j$ if $j$ is within the distance of exposure of hazard scenario $h$ for an event $e$. For every event $e$ relating to an equipment item pair $i, j$, only one non-zero value of $d_{i j h e}^{H}$ is permitted corresponding to a selected hazard scenario from $H_{e}$. These distances are evaluated as shown in equations (B.1) - (B.4) in Appendix B.

For flash fire events, two potential hazard scenarios can occur: HZ1 - where unit $j$ is within the direct reach of the flame envelope and SF - a safe condition where $j$ is outside of the flame envelope produced by unit $i$. To obtain the correct Domino Hazard Score (DHS), the disjunctions in equation (2) are reformulated as shown in equation (63): taking a value of 0 or 10 for a SF and HZ1 scenario respectively:

$$
D H S_{i j, F F}^{e}=10 \cdot B_{i j, H Z 1, F F}^{L} \quad \forall(i, j) \in \Gamma^{H}, j \in I^{V}
$$

where $B_{i j, H Z 1, F F}^{L}$ takes a value of 1 when scenario $H Z 1$ is active for FF events. To ensure that only one of HZ1 or SF scenario occurs, equation (64) is introduced.

$$
\sum_{h \in H_{F F}} B_{i j h, F F}^{L}=1 \quad \forall(i, j) \in \Gamma^{H}, j \in I^{V}
$$

\subsection{Fireball $(F B)$}

For fireball events, three potential hazard scenarios can occur: HZ1 - corresponding to unit $j$ being within direct reach of the flame envelope, FI - corresponding to a HZ1 scenario with a fire insulation installed on the secondary unit and SF a safe condition where unit $j$ is outside of the exposure distance of $i$. The DHS for each hazard scenario, as represented by equation (3), is evaluated using equations (65) - (67):

$$
\begin{array}{cc}
D H S_{i j, F B}^{e}=\sum_{h \in H_{F B}} D H S_{i j h, F B}^{H} & \forall(i, j) \in \Gamma^{H}, j \in I^{A} \\
D H S_{i j, H Z 1, F B}^{H}=10 \cdot B_{i j, H Z 1, F B}^{L} & \forall(i, j) \in \Gamma^{H}, j \in I^{A} \\
D H S_{i j, F I, F B}^{H}=5 \cdot B_{i j, F I, F B}^{L} \quad \forall(i, j) \in \Gamma^{H}, j \in I^{A}
\end{array}
$$


where $H_{F B}=\{H Z 1, F I, S F\}$. A DHS of 10 or 5 is assigned for HZ1 or FI scenarios using equations (66) and (67) respectively. A score of 0 is obtained if neither cases occur, corresponding to a safe (SF) scenario.

The corresponding distances, $d_{i j h, F B}^{H}$, for each hazard scenario are described in equation (B.5) - (B.9) in Appendix B. Finally, only one potential scenario can occur per equipment pair (equation 68), and if a secondary unit $j$ is on a different floor than $i$, the separating floor structure(s) can be assumed to act as a fire insulating material (equation 69)

$$
\begin{gathered}
\sum_{h \in H_{F B}} B_{i j, h, F B}^{L}=1 \quad \forall(i, j) \in \Gamma^{H}, j \in I^{A} \\
B_{i j, F I, F B}^{L}+B_{i j, S F, F B}^{L} \geq 1-N_{i j} \quad \forall(i, j) \in \Gamma^{H}, j \in I^{A}
\end{gathered}
$$

\subsection{Pool fire (PF) and Jet fire (JF)}

For pool and jet fire events, five potential hazard scenarios can occur: HZ1 - corresponding to unit $j$ being within direct reach of the flame envelope, HZ2 - corresponding to unit $j$ being within reach of the effect of heat radiation from the flame envelope, FI - corresponding to a HZ2 scenario with a fire insulation installed on the secondary unit, FW - corresponding to a HZ2 scenario with a firewall installed and SF - a safe condition where unit $j$ is outside of the exposure distance of both the direct flame and radiation effects of $i$.

The DHS for each potential hazard scenario $h$ in a pool fire event, $D H S_{i j h, P F}^{H}$, is evaluated using equations (70) - (76). Each score is calculated based on the separation distance, $d r_{i j, P F}$, between the flame envelope originating in item $i$, and other conditions as outlined in equations (4) and (5). The effective distance for each hazard scenario, $d_{i j h, P F}^{H}$, is obtained using equations (B.10) - (B.26) in Appendix B.

The DHS for pool fire events is the sum of the scores for each potential hazard scenario:

$$
D H S_{i j, P F}^{e}=\sum_{h \in H_{P F}} D H S_{i j h, P F}^{H} \quad \forall i \in I^{V},(i, j) \in \Gamma^{H}
$$

where $H_{P F}=\{H Z 1, H Z 2, F I, F W, S F\}$. For HZ1 scenario to occur, item $j$ has to be within direct reach of the flame envelope corresponding to a DHS of 10:

$$
D H S_{i j, H Z 1, P F}^{H}=10 \cdot B_{i j, H Z 1, P F}^{L} \quad \forall i \in I^{V},(i, j) \in \Gamma^{H}
$$

HZ2 and FI scenarios corresponds to item $j$ being within reach of the effect of heat radiation with no safety device installed. The DHS depends on the nature of the secondary equipment item $j$ as described by Tugnoli et al. (2008). For atmospheric secondary item $j$ :

$$
\begin{aligned}
D H S_{i j h P F}^{H}= & 10 \cdot B_{i j h P F}^{L}-\sum_{\hat{q}} \kappa_{i h, P F, \hat{q}}^{A} \cdot \mu_{i j h, P F, \hat{q}} \\
& \forall i \in I^{V},(i, j) \in \Gamma^{H}, j \in I^{A}, h \in\{H Z 2, F I\}
\end{aligned}
$$


and for pressurized secondary items:

$$
\begin{aligned}
D H S_{i j h, P F}^{H}= & 10 \cdot B_{i j h, P F}^{L}-\sum_{\hat{q}} \kappa_{i h, P F, \hat{q}}^{P} \cdot \mu_{i j h, P F, \hat{q}} \\
& \forall i \in I^{V},(i, j) \in \Gamma^{H}, j \in I^{P}, h \in\{H Z 2, F I\}
\end{aligned}
$$

The value of the piecewise linearisation variable $\mu_{i j h e q}$ is obtained using equations (B.15) - (B.21) based on the separation distance between equipment items.

FW scenarios correspond to the same conditions as the HZ2 scenario with a firewall being installed for item $j$. In such cases a DHS of 1 is assigned:

$$
D H S_{i j, F W, P F}^{H}=B_{i j, F W, P F}^{L} \quad \forall i \in I^{V},(i, j) \in \Gamma^{H}
$$

Where none of these scenarios occur, a DHS of 0 is assigned corresponding to a safe (SF) scenario.

As only one hazard scenario can occur:

$$
\sum_{h \in H_{P F}} B_{i j h, P F}^{L}=1 \quad \forall i \in I^{V},(i, j) \in \Gamma^{H}
$$

Similar to the FB scenario in equation (69), if a secondary unit $j$ is on a different floor, it is assumed that the separating floor structure acts as a fire insulator/wall. Thus:

$$
B_{i j, F W, P F}^{L}+B_{i j, F I, P F}^{L}+B_{i j, S F, P F}^{L} \geq 1-N_{i j} \quad \forall i \in I^{V},(i, j) \in \Gamma^{H}
$$

A similar set of equations apply to a jet fire event and are outlined in Appendix B.3 (equations (B.37) - (B.60)).

\subsection{Blast wave $(B W)$}

With BW events, four potential hazard scenarios can occur for atmospheric secondary equipment items based on the disjunction in equation (7): HZ1 corresponding to unit $j$ being within a distance where static peak overpressure can cause the most damage, HZ2 - corresponding to unit $j$ being within a linearly reducing damage effect of overpressure originating from unit $i, \mathrm{BWl}$ corresponding to a HZ2 scenario with a barricade such as a blast wall (BWl) installed by the secondary unit, and SF - a safe condition where unit $j$ is well outside the distance range of damaging effect from overpressure. For pressurized secondary equipment items, hazard scenario HZ2 does not exist. The evaluation of the distances for each hazard scenario using equations (B.27) - (B.36) in Appendix B.

The DHS for items $i, j$ is the sum of the DHS for each scenario defined by $H_{B W}$ plus an additional term for HZ2 scenario if the secondary item is atmospheric:

$$
D H S_{i j, B W}^{e}=\sum_{h \in H_{B W}} D H S_{i j h, B W}^{H}+\left.D H S_{i j, H Z 2, B W}^{H}\right|_{j \in I^{A}} \quad \forall(i, j) \in \Gamma^{H}
$$


where $H_{B W}=\{H Z 1, B W l, S F\}$. For HZ1 scenario, a DHS of 10 is assigned if the secondary item is within the upper distance limit for pressurized secondary items, and the lower distance limit for atmospheric secondary items.

$$
D H S_{i j, H Z 1, B W}^{H}=10 \cdot B_{i j, H Z 1, B W}^{L} \quad \forall(i, j) \in \Gamma^{H}
$$

HZ2 scenario only occurs for atmospheric secondary equipment items where the DHS is evaluated if the distance from the explosion source is within the upper and lower distance limits $\left(P_{i}^{u p}, P_{i}^{l o}\right)$ :

$$
D H S_{i j, H Z 2, B W}^{H}=\kappa_{e i}^{B W} \cdot d_{i j, H Z 2, B W}^{H}+\lambda_{e i}^{B W} \cdot B_{i j, H Z 2, B W}^{L} \quad \forall(i, j) \in \Gamma^{H} \cap I^{A}
$$

The installation of a barricade such as a blast wall acts as a passive protection measure and limits the effect of overpressure on the secondary item. This corresponds to scenario BWl with a DHS value of 1 :

$$
D H S_{i j, B W l, B W}^{H}=B_{i j, B W l, B W}^{L} \quad \forall(i, j) \in \Gamma^{H}
$$

If an item is at a safe distance away, a DHS value of 0 is obtained.

Finally, only one of the mentioned hazard scenarios can occur for each equipment pair $(i, j)$ depending on the equipment type of the secondary item $j$ - atmospheric or pressurized:

$$
\sum_{h \in H_{B W}} B_{i j h, B W}^{L}+\left.B_{i j, H Z 2, B W}^{L}\right|_{j \in I^{A}}=1 \quad \forall(i, j) \in \Gamma^{H}
$$

Separating floor structures can also act as barricades to reduce the impact of overpressure. Hence, if the two items are on different floors and not within the safe zone, the separating floor is considered to be a blast wall:

$$
B_{i j, B W l, B W}^{L}+B_{i j, S F, P F}^{L} \geq 1-N_{i j} \quad \forall(i, j) \in \Gamma^{H}
$$

\subsection{Protection device cost}

Passive measures to prevent or reduce domino escalation are taken by the installation of protective devices. Each protection device type/configuration $p$ corresponds to a potential hazard scenario which has the possibility of reducing domino effects: fire insulation (FI), firewall (FW), blast wall (BWl). The cost associated with the purchase, installation and maintenance such devices is given as:

$$
C_{i}^{P D}=\sum_{p} C_{i p} \cdot B_{i p} \quad \forall i
$$

where:

$$
\begin{aligned}
B_{j, F I} & \geq B_{i j, F I, e}^{L}+N_{i j}-1 \quad(i, j) \in \Gamma^{H}, e \\
B_{j, F W} & \geq B_{i j, F W, e}^{L}+N_{i j}-1 \quad(i, j) \in \Gamma^{H}, e \\
B_{j, B W l} & \geq B_{i j, B W l, B W}^{L}+N_{i j}-1 \quad(i, j) \in \Gamma^{H}
\end{aligned}
$$

The last two terms on the RHS of equations (84) - (86) ensure that passive protection device cost are included only when such device is installed, as opposed to separating floors acting as firewalls, fire insulators and/or blast walls. 


\subsection{Expected losses cost}

The total cost attributed to losses caused by accidental events in a unit $i$ is given by the Domino Escalation Cost $C_{i}^{D E C}$.

$$
\begin{gathered}
C_{i}^{D E C}=\sum_{j:(i, j) \in \Gamma^{H}} C_{j}^{A L} \cdot C r_{i j} \quad \forall i \\
C_{j}^{A L}=C_{j}^{P} \quad \forall j
\end{gathered}
$$

It accounts for the loss associated with domino escalation to secondary units, and $C_{j}^{A L}$ is the direct asset loss of the unit. This direct asset loss is represented as the purchase cost of all the equipment items affected. $C r_{i j}$ is the parameter that represents the assurance that an event in item $i$ affects secondary item $j$. It is determined as a function of the Domino Hazard Score $\left(D H S_{i j}\right)$. This function is expressed as (de Lira-Flores et al., 2014):

$$
C r_{i j}=a \cdot D H S_{i j}^{3}+b \cdot D H S_{i j}^{2}+c \cdot D H S_{i j} \quad \forall(i, j) \in \Gamma^{H}
$$

where $a=6.7374 \times 10^{-4}, b=4.9158 \times 10^{-4}$ and $c=2.7498 \times 10^{-2}$.

The human health loss and environmental clean-up cost are not considered.

Equation (89) has non-linear terms hence a piecewise linear approximation is used (D'Ambrosio et al., 2010). Samples points, $\hat{k}$, are taken for $D H S_{i j}$ between 0 and $10\left(D \hat{H} S_{i j \hat{k}}\right)$ and the corresponding values of $C r_{i j}$ evaluated $\left(\hat{C} r_{i j \hat{k}}\right)$. A SOS2 variable $\left(\phi_{i j \hat{k}}\right)$ is introduced and equation (89) is re-written as:

$$
\begin{aligned}
C r_{i j} & =\sum_{\hat{k}} \hat{C} r_{i j \hat{k}} \phi_{i j \hat{k}} \quad \forall(i, j) \in \Gamma^{H} \\
D H S_{i j} & =\sum_{\hat{k}} D \hat{H} S_{i j \hat{k}} \phi_{i j \hat{k}} \quad \forall(i, j) \in \Gamma^{H}
\end{aligned}
$$

\subsection{Objective function}

The objective function is then to minimize the total cost attributed to connection, pumping, construction, protection device installation and domino escalation:

$$
\begin{array}{r}
\min \sum_{(i, j) \in \Gamma^{C}}\left(C_{i j}^{C} T D_{i j}+C_{i j}^{V} D_{i j}+C_{i j}^{H}\left(R_{i j}+L_{i j}+A_{i j}+B_{i j}\right)\right) \\
+F C 1 \cdot N F+F C 2 \sum_{s} A R_{s} \cdot N Q_{s}+L C \cdot F A \\
+\sum_{i} C_{i}^{P D}+\sum_{i} C_{i}^{D E C}
\end{array}
$$

subject to layout constraints (14) - (22), (A.1) - (A.3), (A.5) - (A.35); and safety constraints $(1),(12),(13),(23)$ - (28), (33) - (35), (37) - (47), (49) - (88), (90) - 
(91) and (B.1) - (B.60). This constitutes model DHI_MILP. The total ${ }^{1}$ number of variables for model DHI_MILP can be obtained as follows:

$$
\begin{array}{r}
\begin{array}{r}
\text { No. of binary variables }=|\hat{S}|+|K|+|I|(49|I|+0.5|I||K|+1.5|K|-45) \\
\\
-\left.0.5|K|\left(|I|-\left|I^{T}\right|\right)\left(|I|-\left|I^{T}\right|-1\right)\right|_{\left(|I|-\left|I^{T}\right|\right)>1} \\
\text { No. of continuous variables }=\left|I^{T}\right|+|\hat{S}|+|I|(91|I|-84)+14
\end{array}
\end{array}
$$

\section{Case Study}

The proposed model was applied to an acrylic acid production (AA) plant (Figure 6) adapted from de Lira-Flores et al. (2014). The plant consists of 11 equipment items with details on equipment dimensions, costs and connectivity are available in the Appendix C. Values for the damage index, equipment purchase cost, protection device cost, and other safety data are given in Table C.2, and four floors were made available for layout considerations. The example was solved to global optimality with and without safety considerations using models DHI_MILP and OPTL (Appendix A) respectively. For model OPTL, the domino escalation cost was post processed based on the same considerations in model DHI_MILP. GAMS (GAMS Development Corporation, 2018) modelling system v25.0.2 with CPLEX v12.8.0.0 solver was used on an Intel ${ }^{\circledR}$ Xeon ${ }^{\circledR}$ E5$1650 \mathrm{CPU}$ having 12 threads with 32GB RAM.

Highly hazardous equipment item pairs $\left(\Gamma^{H}\right)$ were identified using the values in Table C.2 and equation (1). The acid extractor (item 7) and solvent mixer (item 9) were identified as atmospheric equipment items $\left(I^{A}\right)$ and also prone to flammable vapours $\left(I^{V}\right)$ having a radius of exposure for pool fire events of $3.6 \mathrm{~m}$ and $4.0 \mathrm{~m}$ respectively.

\footnotetext{
${ }^{1}$ This represents the maximum values equivalent to a worst case scenario where all equipment items are connected to each other, and all possible pairs are considered to be highly hazardous and prone to all considered events
} 


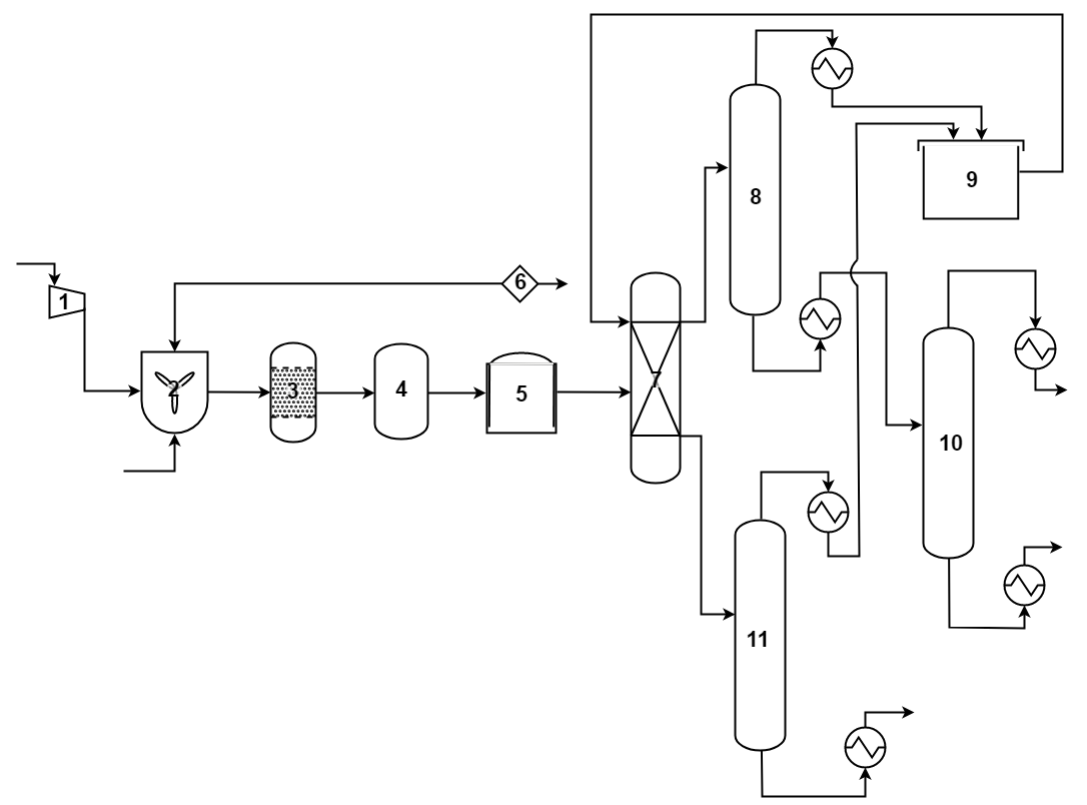

Figure 6: Flow diagram of acrylic acid process plant (See Table C.1 in the Appendix for a description of the equipment item labels)

\subsection{Layout results}

Table 2 gives a summary of the model statistics for each of the runs performed - with and without safety considerations. In both considerations, the models obtained the globally optimal solution in less than six minutes. Despite DHI_MILP having a greater number of equipment items (when compared to similar considerations in literature) and a relatively larger number of decision variables, the solution was obtained in 307.3s. This proves a higher model efficiency.

Table 2: Model Statistics \& computational performance

\begin{tabular}{lcc}
\hline & Without safety & With safety \\
\hline CPU (s) & 7.0 & 307.3 \\
Number of discrete variables & 303 & 1578 \\
Number of continuous variables & 381 & 2131 \\
Number of equations & 1101 & 4928 \\
\hline
\end{tabular}

Figures 7 and 8 show the optimal layout of the AA plant without and with safety considerations, each having a total cost of 789,159.1 rmu and 667,112.7 rmu respectively as shown in Table 3 . A greater number of floors was required (4 floors) when safety was considered compared to 3 floors without safety constraints, each measuring $5 \mathrm{~m} \times 10 \mathrm{~m}$. This additional floor allowed for a reduced risk level. Items 7 and 9 , which were particularly prone to more hazardous events than 
the others, were spaced from most of the other equipment items in floors 2 and 1 respectively. Their location on different floors also allowed for the separating floor structure to act as a passive protection device. Item 5 (floor 3) was placed close to item 7 though as the equipment item pair was not identified as hazardous based on the criteria in equation 1. As such, the model accounts for specific interactions between equipment items based on the DHI rules to ensure minimal risk.

Table 3: Cost breakdown

\begin{tabular}{lcc}
\hline & Without safety & With safety \\
\hline Layout cost $(\mathrm{rmu})$ & $617,619.3$ & $652,148.3$ \\
Domino escalation cost $(\mathrm{rmu})$ & $171,539.8$ & $12,613.3$ \\
Safety device cost $(\mathrm{rmu})$ & 0.0 & $2,351.0$ \\
Total cost $(\mathbf{r m u})$ & $\mathbf{7 8 9 , 1 5 9 . 1}$ & $\mathbf{6 6 7 , 1 1 2 . 7}$ \\
\hline
\end{tabular}

This minimization of risk by the current layout is captured by the Domino escalation cost. Although a larger layout cost $(652,148.3$ vs $617,619.3 \mathrm{rmu})$ was obtained when safety was considered, a much greater reduction is achieved in terms of the financial risk $(12,613.3 \mathrm{vs} 171,539.8 \mathrm{rmu})$. Layout costs encompass the costs of piping, pumping, purchasing land and constructing the selected floors, while the Domino escalation cost deals with the purchase cost of all secondary units affected by the primary items event(s). Although not currently quantified, the later cost also affects plant personnel and the neighbouring environment by extension.

Floor $1 / 3$

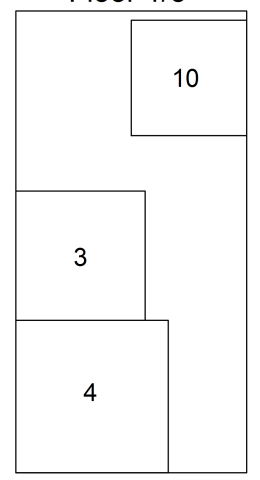

Floor 2/3

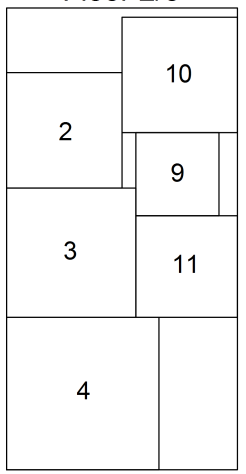

Floor 3/3

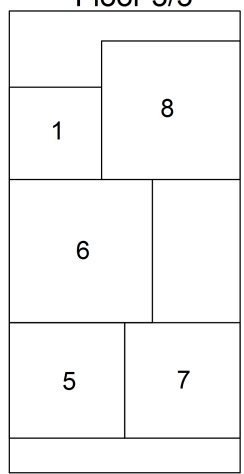

Figure 7: Optimal layout of A A Plant without safety considerations 


\section{Floor $1 / 4$}

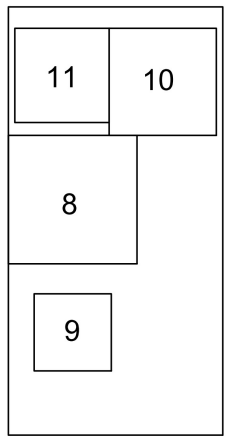

Floor $3 / 4$

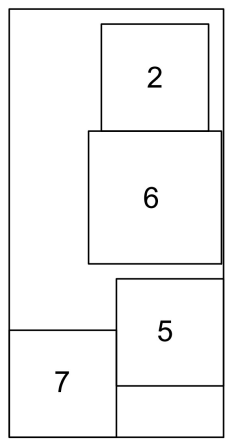

Floor $2 / 4$

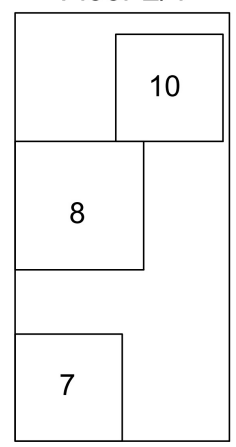

Floor $4 / 4$

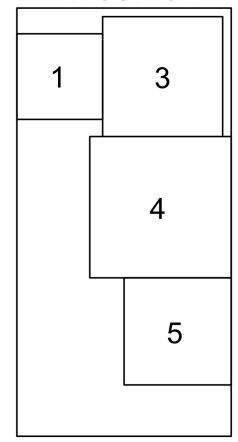

Figure 8: Layout of AA Plant with safety considerations

Table 4 shows the DHI values for each equipment item with and without safety considerations, as well as the units with installed safety devices for the former case. The DHI value is the sum of scores given to the worst possible event that can occur on all secondary units, escalating from a primary unit. A score of 0 indicates an inherently 'safer' level while a higher score indicates a greater probability for domino escalation. Across all units, Table 4 shows a reduction in the non-zero DHI values. This denotes a general increase in the overall safety levels of the plant. For some equipment items, protection devices were installed to ensure this - fire insulation on item 11. New FWs and BWls were not purchased primarily because the separating floor structure was assumed to act as such. Hence, the need to purchase was prevented as the solution, in most cases, placed such hazardous item pairs on separate floors or outside the radius of exposure of the primary item e.g., primary item 1 and items 2 and 7 may have required a blast wall if they were closer together and on the same floor. 
Table 4: DHI values \& installed protection devices on equipment items

\begin{tabular}{clccccc}
\hline$i$ & Description & DHI $^{1}$ & DHI $^{2}$ & FI & FW & BWl \\
\hline 1 & Compressor & 0.0 & 0.0 & & & \\
2 & Feed mixer & 0.0 & 0.0 & & & \\
3 & Reactor & 0.0 & 0.0 & & & \\
4 & Quench & 0.0 & 0.0 & & & \\
5 & Absorber & 0.0 & 0.0 & & & \\
6 & Splitter & 10.0 & 0.0 & & & \\
7 & Acid Extractor & 1.0 & 1.0 & & & \\
8 & Distillation 1 & 0.0 & 0.0 & & & \\
9 & Solvent mixer & 32.0 & 5.5 & & & \\
10 & Distillation 2 & 0.0 & 0.0 & & & \\
11 & Distillation 3 & 10.0 & 0.0 & $\checkmark$ & & \\
\hline
\end{tabular}

${ }^{1}$ Without safety considerations

${ }^{2}$ With safety considerations

\subsection{Distance metric comparison}

As stated earlier, the Tchebychev distance was used to calculate the separation distance between equipment items for safety considerations. This metric was adopted as it represented a better approximation to the Euclidean distance when compared to rectilinear measurements. The Euclidean distance, although a more accurate measurement, introduces non-linear terms to the model, making it difficult to and/or unpredictable in obtaining solutions for different problems. To demonstrate the benefits of this choice of the Tchebychev metric, the DHI value for each unit was post-processed using the results obtained in the final layout with safety considerations, recalculating the separation distances from the equipment boundaries using the Euclidean distance metric. The DHI values were chosen as they have a direct effect on the overall safety level, choice of protection devices and layout configuration of the plant. The comparison is shown in Table 5.

For all equipment items shown in Table 5, the DHI values calculated using the Tchebychev metric matches those obtained using the Euclidean distance. Hence, the Tchebychev distance is well suited for estimating the safety/separation distance without the associated computational complexity when using the Euclidean metric. 
Table 5: Distance metric comparison and the effect on DHI values

\begin{tabular}{clcc}
\hline \multirow{2}{*}{ Equipment } & \multirow{2}{*}{ Description } & \multicolumn{2}{c}{$D H I_{i}$} \\
& & Tchebychev & Euclidean \\
\hline 1 & Compressor & 0.0 & 0.0 \\
2 & Feed mixer & 0.0 & 0.0 \\
3 & Reactor & 0.0 & 0.0 \\
4 & Quench & 0.0 & 0.0 \\
5 & Absorber & 0.0 & 0.0 \\
6 & Splitter & 0.0 & 0.0 \\
7 & Acid Extractor & 1.0 & 1.0 \\
8 & Distillation 1 & 0.0 & 0.0 \\
9 & Solvent mixer & 5.5 & 5.5 \\
10 & Distillation 2 & 0.0 & 0.0 \\
11 & Distillation 3 & 0.0 & 0.0 \\
\hline
\end{tabular}

\subsection{Exploring sub-optimal solutions}

The results presented in the previous sections have focused solely on the globally optimal solution of the proposed model. This solution presents the most cost effective layout design based on the considerations of the model. In practice however, additional considerations are bound to be explored which are unique to the decision maker, project and/or region of application. It then becomes important to understand common features, 'must haves' (Voll et al., 2015), and patterns amongst the near-optimal solutions.

To obtain this, model DHI_MILP ${ }^{\mathrm{s}}$ is solved iteratively after the globally optimal solution is obtained from model DHI_MILP. Results obtained will be optimal solutions with increasing total cost values. DHI_MILPs ${ }^{\mathrm{s}}$ constitutes model DHI_MILP with the inclusion of an integer cut (equation 93) to exclude previously obtained solutions.

$$
\begin{array}{r}
\sum_{(i, j) \in U_{i j v \tau}^{E 1}} E 1_{i j}+\sum_{(i, j) \in U_{i j v \tau}^{E 2}} E 2_{i j}-\sum_{(i, j) \in L_{i j v \tau}^{E 1}} E 1_{i j}-\sum_{(i, j) \in L_{i j v \tau}^{E 2}} E 2_{i j} \\
\leq\left|U_{i j v \tau}^{E 1}\right|+\left|U_{i j v \tau}^{E 2}\right|-1 \quad \forall v, \tau
\end{array}
$$

where $v$ is the optimal solution count, $\tau$ is the symmetry (reflection and/or rotation) of the optimal solution, and:

$$
\begin{aligned}
& L_{i j v \tau}^{E 1}=\left\{(i, j): j>i, E 1_{i j}=0, N_{i j}=1 ; \forall v, \tau\right\} \\
& U_{i j v \tau}^{E 1}=\left\{(i, j): j>i, E 1_{i j}=1, N_{i j}=1 ; \forall v, \tau\right\} \\
& L_{i j v \tau}^{E 2}=\left\{(i, j): j>i, E 2_{i j}=0, N_{i j}=1 ; \forall v, \tau\right\} \\
& U_{i j v \tau}^{E 2}=\left\{(i, j): j>i, E 2_{i j}=1, N_{i j}=1 ; \forall v, \tau\right\}
\end{aligned}
$$

It should be noted that for each solution obtained by solving the model, there exists at least 7 other possible alternative solutions which are reflections 
and/or rotations of it, with the same objective function value. These symmetric solutions are illustrated in Figure 9 . Key binary variables $\left(O_{i}, E 1_{i j}, E 2_{i j}\right)$ for such solution are saved $(\tau=1)$, and in order to save computational time, the symmetric solutions are manually generated by appropriately changing the $O_{i}, E 1_{i j}, E 2_{i j}$ binary variables. A typical example of how these binary variables change over each symmetric solution is illustrated in Table 6 for the example in Figure 9. Hence, for each loop the model is solved excluding previous solutions and their reflected/rotated versions.

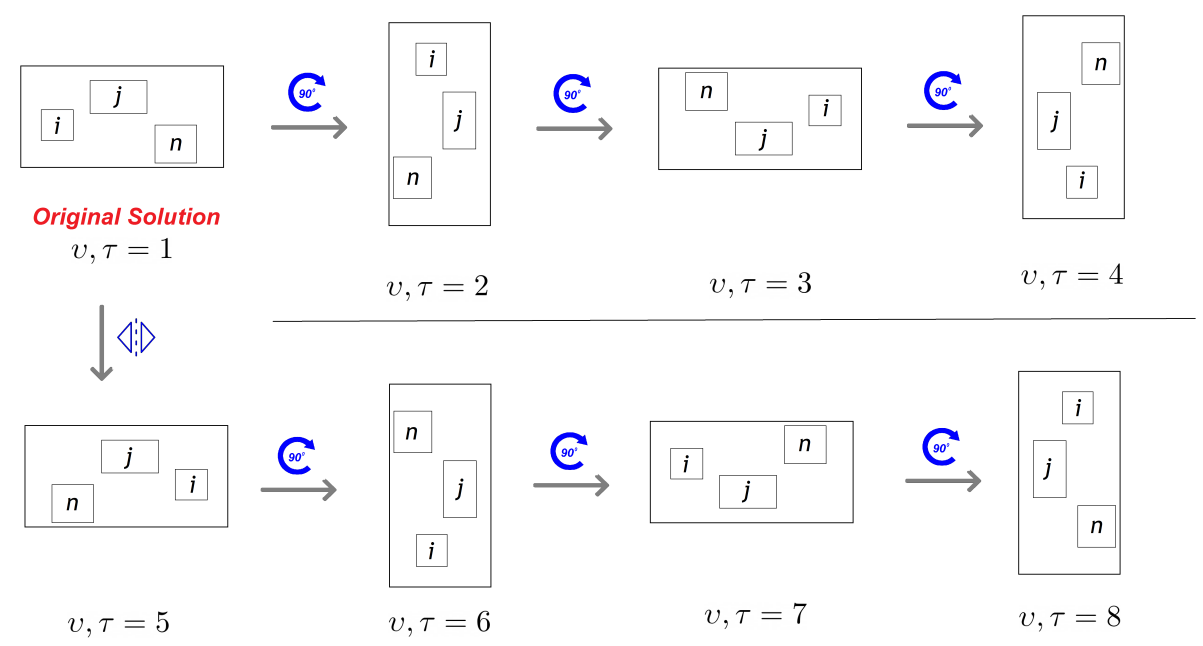

Figure 9: Symmetric solution illustration

Table 6: Typical example of key binary variable changes in symmetric solutions

\begin{tabular}{ccccc|cccc}
\hline$\tau$ & $E 1_{i j}$ & $E 2_{i j}$ & $O_{i}$ & $O_{j}$ & $E 1_{j n}$ & $E 2_{j n}$ & $O_{j}$ & $O_{n}$ \\
\hline 1 & 1 & 1 & 1 & 0 & 1 & 0 & 0 & 0 \\
2 & 1 & 0 & 0 & 1 & 0 & 0 & 1 & 1 \\
3 & 0 & 0 & 1 & 0 & 0 & 1 & 0 & 0 \\
4 & 0 & 1 & 0 & 1 & 1 & 1 & 1 & 1 \\
5 & 0 & 1 & 1 & 0 & 0 & 0 & 0 & 0 \\
6 & 1 & 1 & 0 & 1 & 0 & 1 & 1 & 1 \\
7 & 1 & 0 & 1 & 0 & 1 & 1 & 0 & 0 \\
8 & 0 & 0 & 0 & 1 & 1 & 0 & 1 & 1 \\
\hline
\end{tabular}

Figure 10 shows the landscape-oriented layout plots of optimal solutions $(\mathrm{Rx})$ ordered by increasing total cost value. The total layout cost values are indicated and their corresponding total DHIs. Major changes to the position of equipment items in each plot compared to the globally optimum plot (R1) are 
shaded red. Changes are considered major when an equipment item changes the floor it was located on and its position within the floor. Minor changes in floor $x, y$ positions were neglected. Solutions 1 through 4 are shown in the Figure 10.
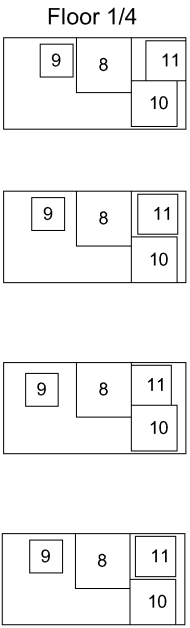
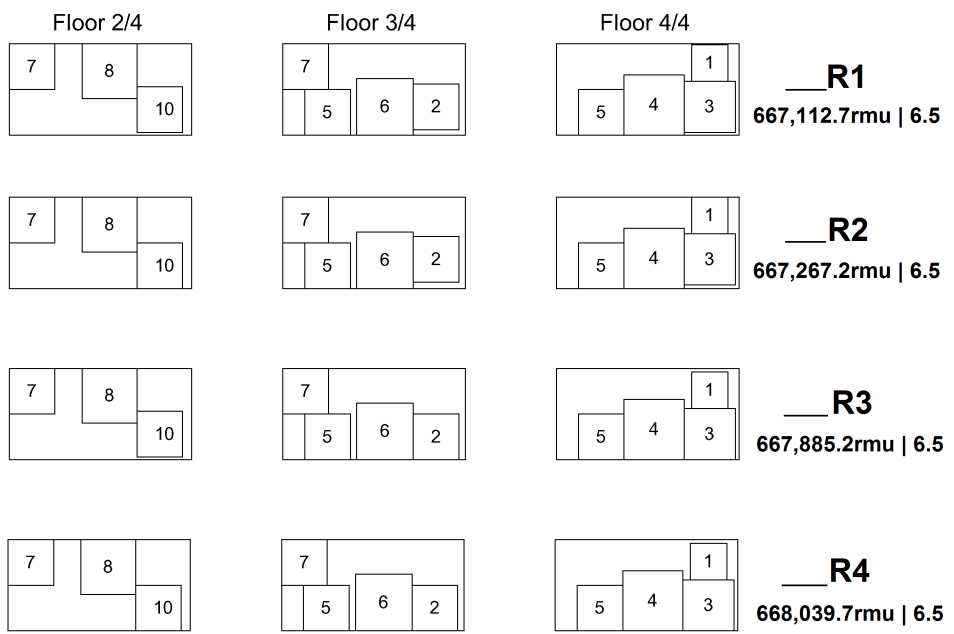

Figure 10: Optimal solutions: Layout plots, total cost and total DHI

It can be seen that although there are changes in the total cost value across the solutions, only minor changes occur in the actual layout configuration. These changes occur with items 10 and 11 having varying $\mathrm{x}, \mathrm{y}$ positions across solutions. There are also no changes to the total DHI values across solutions and hence the safety levels. Though these observations, the findings are still useful to a decision maker as it shows how sensitive the layout configuration is to the total cost. Where additional features or changes need to be incorporated later on, the decision maker is more informed on how flexible the layout configuration is and its impact on the total cost.

In some other cases, the decision maker may not be as cost-conscious but desires alternative layout configurations with much more significant changes. In such situation, alternative solutions with a great degree of variation can be obtained by replacing equation (93) with (94) in model DHI_MILP':

$$
\sum_{(i, j) \in U_{i j v \tau}^{N}} N_{i j}-\sum_{(i, j) \in L_{i j v \tau}^{N}} N_{i j} \leq\left|U_{i j v \tau}^{N}\right|-1 \quad \forall v, \tau
$$

where:

$$
\begin{aligned}
& L_{i j v \tau}^{N}=\left\{(i, j): j>i, N_{i j}=0 ; \forall v, \tau\right\} \\
& U_{i j v \tau}^{N}=\left\{(i, j): j>i, N_{i j}=1 ; \forall v, \tau\right\}
\end{aligned}
$$

Figure 11 shows the layout plots of the new set of optimal solutions $(\mathrm{Rx})$. The solutions are ordered by increasing total cost value and show a greater 
degree of variation in layout configuration. Through solutions R1 - R4, items 4, 5, 7 and 9 have major variations in floor location and position, with other items only varying in $\mathrm{x}, \mathrm{y}$ positions on the same floor. Changes in the total DHI values are also observed unlike the previous case, with $\mathrm{R} 1$ still having the highest safety level. A larger difference in total cost between solutions is also observed, but these set of solutions are more beneficial were disparate layout configurations are sort by the decision maker, with less concern for the costs.
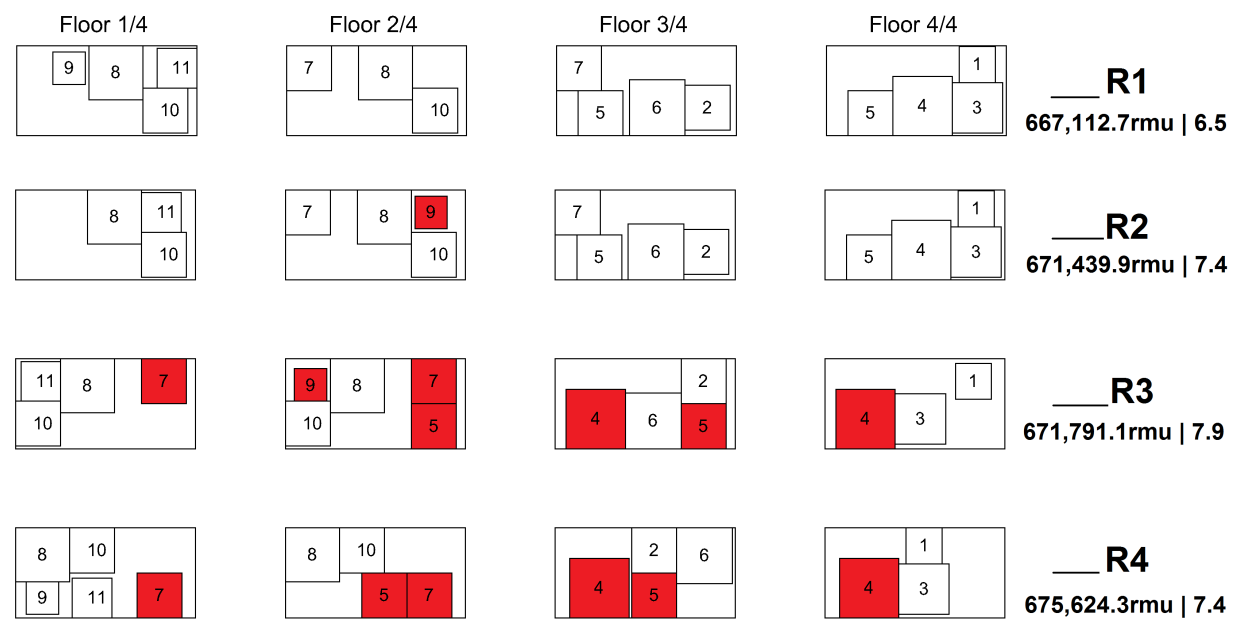

Figure 11: Optimal solutions 2: Layout plots, total cost and total DHI

\subsection{Multi-objective considerations}

It also becomes important to ensure that plant safety levels are kept below certain pre-defined industry standards. As such, solving for the minimum total cost (layout and safety) is insufficient as such thresholds cannot be guaranteed. In such cases, understanding the financial implications for specific safety thresholds becomes important. This requires a multi-objective study.

Two metrics can be analysed: the total monetary $\operatorname{cost}\left(f^{C}\right)$ and the total DHI $\left(f^{S}\right)$. The total DHI value gives an indication of the safety levels for the layout configuration of the plant. The $\epsilon$-constraint method (Haimes et al., 1971) is adopted to solve this problem. Generally, the multi-objective optimisation problem comprising a number of objective functions is solved using the $\epsilon$ constraint method by converting all but one objective into constraints, setting upper (or lower) bounds to them. This method has been identified as probably the best known technique for multi-criteria optimisation problems (Chiandussi et al., 2012) and has been used in literature for similar problems (Almaraz et al., 2016, Liu and Papageorgiou, 2013, Zhang et al., 2016). In the current case with two objectives $\left(\min \left\{f^{C}, f^{S}\right\}\right)$, the multi-objective problem is reformulated as: 


$$
\begin{aligned}
\min & f^{C} \\
\text { s.t. } & f^{S} \leq \epsilon^{S}
\end{aligned}
$$

where:

$$
\begin{array}{r}
f^{C}=\sum_{(i, j) \in \Gamma^{C}}\left(C_{i j}^{C} T D_{i j}+C_{i j}^{V} D_{i j}+C_{i j}^{H}\left(R_{i j}+L_{i j}+A_{i j}+B_{i j}\right)\right) \\
+F C 1 \cdot N F+F C 2 \sum_{s} A R_{s} \cdot N Q_{s}+L C \cdot F A \\
+\sum_{i} C_{i}^{P D}
\end{array}
$$

Values of $\epsilon^{S}$ ranging from 0 - 55 with a step size of 5 were used to obtain a set of optimal solutions. Additionally, $\epsilon^{S}$ values in the range of $5.0-6.5$ were used with a step size of 0.5 to capture the trend near the single-objective optimal solution from section 5.1.

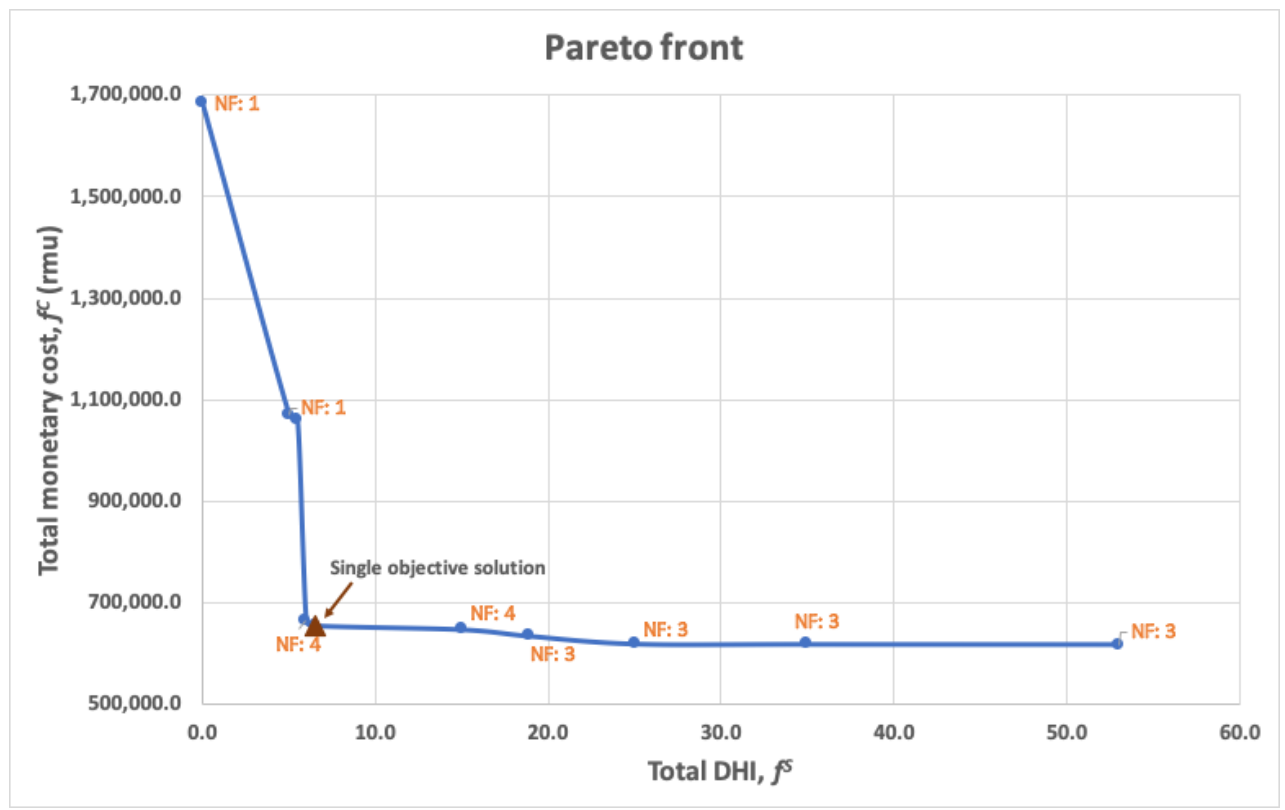

Figure 12: Pareto front for multi-objective consideration

Figure 12 shows a plot of the pareto-optimal solutions for the total monetary cost of the plant and total DHI values, with indication of the number of optimal 
floors for each solution. As expected, a reduction in the maximum total DHI value in each run led to an increase in cost up to a maximum value of $1,683,128.0$ rmu. This value represents the monetary cost of a layout configuration with the least possibility for domino escalation either by the various types of fires or overpressure.

A relatively greater increase in layout costs is also observed for a reduction in total DHI values between 18.9 and 15.0, when compared to previous changes. This is attributed to an additional floor requirement leading to an increase in construction costs. More significant changes in total cost only occur below a total DHI value of 6.0. This shows that a large reduction in the domino escalation potential of the plant can be achieved with little or no change to the layout configuration (and cost), down to a total DHI value of 6 . Additional changes to increase safety levels within the plant lead to a relatively larger monetary commitment.

Figure 13 gives a breakdown of the costs for each pareto-optimal solution the total monetary cost (connection, pumping, construction and safety device installation cost) and the domino escalation cost. As the domino escalation cost directly relates to the safety levels of the plant, its value reduces as the total DHI value is lowered. The construction and pumping costs take a significant share of the total monetary cost. Construction costs values are constant down to a total DHI value of 18.9 , with increments only in the connection and pumping costs. This hints to a rearrangement of intra-floor items towards a more sparse layout configuration and proves that reduced risk levels within a plant can be achieved, in most instances, without significant cost implications.

An increase in the optimal number of floors from 3 to 4 for total DHI values below 18.9, down to 6.0, led to an increase in construction cost. This is due to the inclusion of an additional floor as the floor area remains the same $(5 \mathrm{~m}$ $\times 10 \mathrm{~m})$. As stated previously, separating floor structures are assumed to act as passive protection devices, thus contributing to a reduced total DHI value. For solutions with a total DHI value below 6.0, although the optimal solutions required a single floor, a wider floor area $(5 \mathrm{~m} \times 25 \mathrm{~m})$ was required, increasing in length up to the minimum total DHI value.

Hence, from the point of view of monetary cost, solutions with a total DHI value in the range of $6.0-18.9$ are recommended, if allowed by safety requirements. Note that the decision-maker may still select any other optimal solution on the pareto-front to meet the required safety standards for the plant.

\section{Concluding remarks}

In this work, an efficient MILP model was proposed for the multi-floor process plant layout problem considering the domino effect of hazardous events on process plant equipment items. Using the set of rules outlined by the domino hazard ranking system, primary equipment items susceptible to hazardous events such as flash fires, jet fires, pool fires, fire balls and blast waves were identified, with secondary items which could also be affected. Using the DHSs, the probability (and impact) of these incidents escalating to secondary items were estimated 


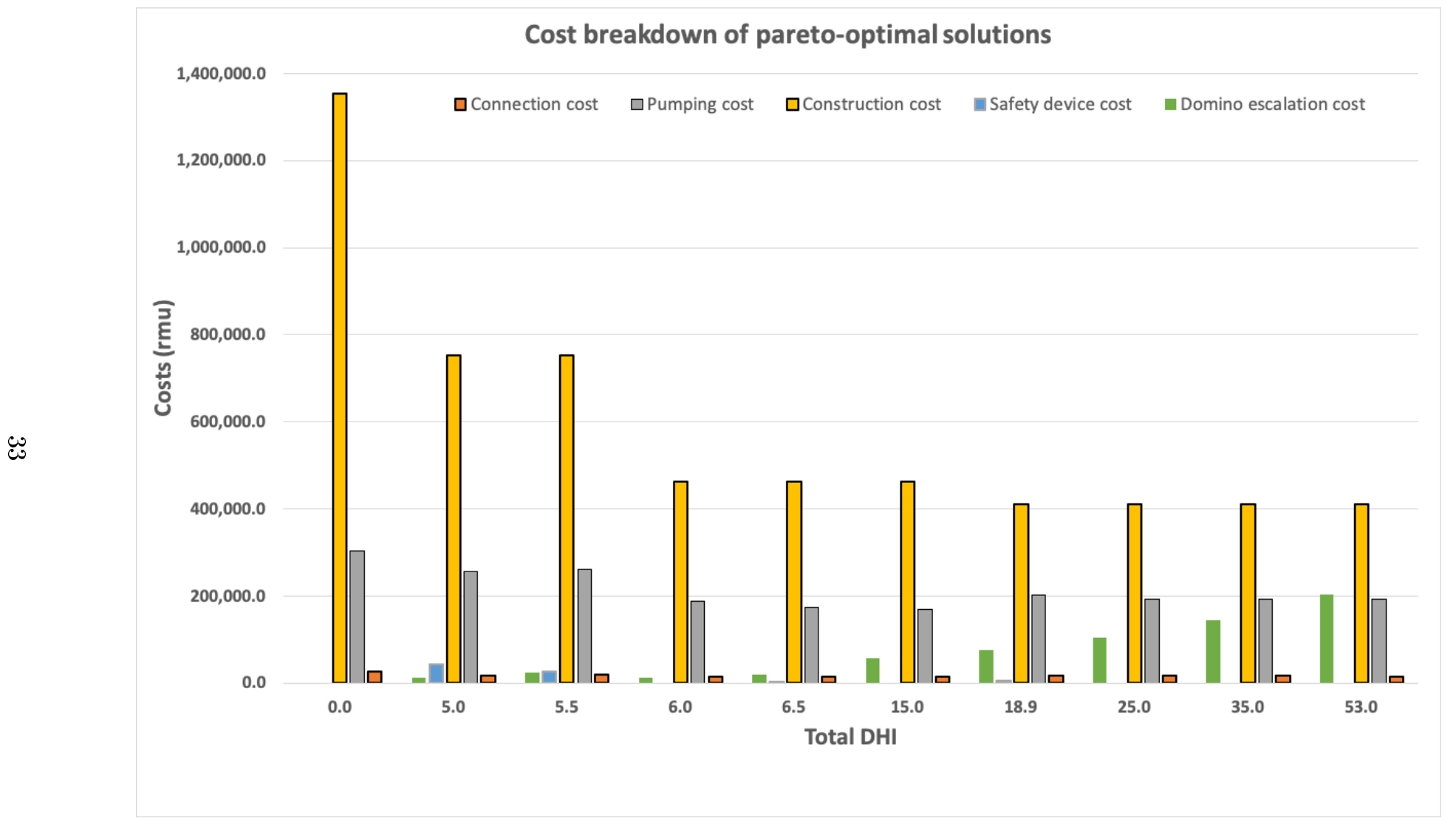

Figure 13: Cost breakdown of Pareto-optimal solutions 
and included in an MILP model with an objective to simultaneously minimise the total costs attributed to the layout of the equipment item, losses due to equipment damage as well as the installation of protection devices to mitigate such events. Multi-objective considerations of layout costs and total DHI values were also explored.

With an acrylic acid plant from literature as a case study, the performance and capability of the proposed model were demonstrated. Globally optimal solutions were obtained in less than six minutes with all aforementioned incidents of fires and blast wave being accounted for. Layout configurations showed that hazardous equipment items were placed outside of the primary item's radius of exposure or a protection device was installed. Although the total layout cost was higher when compared to the layout configuration without safety consideration, the domino escalation cost savings were far greater. Sub-optimal solutions were also explored. Using integer cuts, multiple solutions ordered by increasing total cost values were obtained and patterns amongst optimal solutions were identified towards a more flexible approach for optimal layout configurations. Bi-objective considerations were also explored. Using the $\epsilon$-constraint method, a set of pareto-optimal solutions were obtained. Results showed that the layout configuration with the least possibility for domino escalation corresponded to a cost value of $1,683,128.0 \mathrm{rmu}$, and a total DHI value in the range of $6.0-18.9$ provided the optimal balance of layout costs and safety levels within the plant.

For future research, additional escalation vectors need to be explored in conjunction with those considered in this work. Scenarios of toxic release and the combined effect on humans within and outside the plant area are worthy of note. Uncertainty considerations for safety and cost parameters may also be explored to ensure a more robust solution for process plants.

\section{Acknowledgement}

JOE gratefully acknowledges the Petroleum Technology Development Fund (PTDF), Nigeria; Peter Lai and Christopher Thong for their input during the early model development phase. SL is supported by the Fundamental Research Funds for the Central Universities (AUGA5710050220).

\section{Nomenclature}

The abbreviations and symbols used are defined as follows:

\section{Abbreviations}

$\begin{array}{ll}\text { BW } & \text { Blast wave } \\ \text { BW1 } & \text { Blast wall } \\ \text { DHI } & \text { Domino Hazard Index } \\ \text { FB } & \text { Fire ball } \\ \text { FF } & \text { Flash fire }\end{array}$


FI

FW

HZ1

HZ2

JF

PF

SF

Indices

$\theta$

$\tau$

$v$

e

$h$

$i, j, n$

$k$

$p$

s

$\hat{k}$

$\hat{q}$

Sets

$\Gamma^{C}$

$\Gamma^{H}$

E

$H_{e}$

I

$I^{A}$

$I^{T}$

$I^{P}$

$I^{V}$

K

$\hat{S}$

Parameters
Fire insulation

Firewall

Potential hazard scenario 1

Potential hazard scenario 2

Jet fire

Pool fire

Safe scenario

floor count index

reflections and/or rotations of optimal solution

optimal solution number

primary events/scenarios

potential hazard scenarios

equipment item

floor number

protection devices

rectangular area sizes

piecewise linearisation sample points for $C r_{i j}$ variable

piecewise linearisation sample points for pool and jet fire $D H S_{i j}$ variable

set of ordered pairs of connected items $i$ and $j$

set of ordered pairs of items $i$ and $j$ regarded as highly hazardous

set of primary events/scenarios; $E=\{F F, F B, J F, P F, B W\}$

set of potential hazard scenarios for primary event $e ; \bigcup_{e} H_{e}=$

$\{H Z 1, H Z 2, F I, F W, B W l, S F\}$

set of equipment items

set of atmospheric equipment items

set of tall/multi-floor equipment items

set of pressurised equipment items

set of equipment items likely to release flammable vapours

set of potential floors

set of predefined rectangular area dimensions
$\alpha_{i}, \beta_{i}, \gamma_{i}$
dimensions of equipment item $i$
$\delta_{i \theta}$
1 for equipment item $i$ if $\theta \leq I^{T} ; 0$ otherwise
a very small positive number 


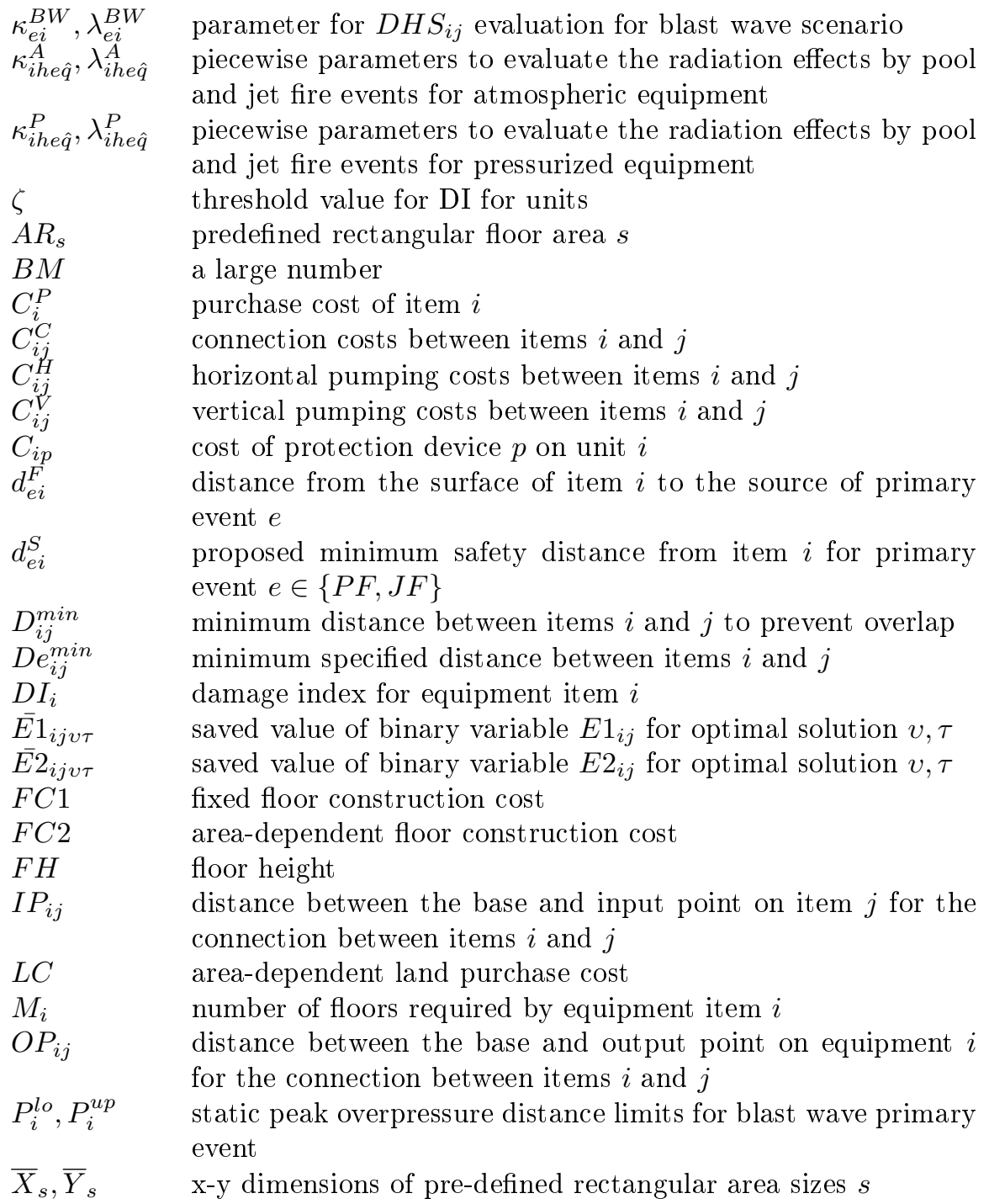

Integer variables

NF number of floors

Binary variables

$\begin{array}{ll}B_{i p} & 1 \text { if protective device } \mathrm{p} \text { is installed on equipment item } i 0, \\ & \text { otherwise } \\ B_{i j h e \hat{q}}^{F} & 1 \text { interval } \hat{q} \text { is selected to evaluate the radiation effects by pool } \\ & \text { and jet fire events; 0, otherwise }\end{array}$ 


$\begin{array}{ll}B_{i j h e}^{L} & \begin{array}{l}1 \text { if secondary unit } j \text { is located within the boundaries for hazard } \\ \text { scenario } h \text { owing to an event } e \text { emanating from item } i\end{array} \\ E 1_{i j}, E 2_{i j} & \begin{array}{l}\text { non-overlapping binary, a set of values which prevents } \\ \text { equipment overlap in one direction in the x-y plane }\end{array} \\ M B_{i j}^{X Y} & 1 \text { if } X D_{i j} \geq Y D_{i j} ; 0 \text { otherwise } \\ M B_{i j}^{Z} & 1 \text { if } X Y_{i j}^{m a x} \geq V D_{i j} ; 0 \text { otherwise } \\ N_{i j} & 1 \text { if items } i \text { and } j \text { are assigned to the same floor; } 0 \text {, otherwise } \\ N_{i j k}^{\prime} & 1 \text { if items } i \text { and } j \text { are assigned to floor } \mathrm{k} \text {; otherwise } \\ O_{i} & 1 \text { if length of item } i \text { is equal to } \alpha_{i} ; 0, \text { otherwise } \\ Q_{s} & 1 \text { if rectangular area } s \text { is selected for the layout; } 0, \text { otherwise } \\ S_{i k}^{S} & 1 \text { if item } i \text { begins on floor } k ; 0, \text { otherwise } \\ V_{i k} & 1 \text { if item } i \text { is assigned to floor } k \\ W_{k} & 1 \text { if floor } k \text { is occupied; } 0, \text { otherwise } \\ W_{i j}^{X} & 1 \text { if item } i \text { is to the right of item } j \text { in the x plane; } 0 \text { otherwise } \\ W_{i j}^{X} & 1 \text { if the boundary of item } i \text { is strictly to the right or left of item } \\ W_{i j}^{Y} & j \text { in the x plane; } 0 \text { otherwise } \\ W_{i j}^{Y O} & 1 \text { if item } i \text { is above item } j \text { in the y plane; } 0 \text { otherwise } \\ W_{i j}^{Z} & 1 \text { if the boundary of item } i \text { is strictly above or below item } j \text { in } \\ & \text { the y plane; } 0 \text { otherwise }\end{array}$

\section{Continuous variables}

$\begin{array}{ll}\eta_{i j}^{U}, \eta_{i j}^{D} & \text { vertical safety distance between items } i \text { and } j \\ \mu_{i j h e \hat{q}} & \text { variable for selection of piecewise parameter to evaluate } \\ & \text { radiation effects of pool and jet fire events } \\ \omega_{i} & \text { number of floors by which a multi-floor item } i \in I^{T} \text { extends } \\ & \text { over the topmost floor } \\ \phi_{i j \hat{k}} & \text { variable for piecewise linearisation of } C r_{i j} \text { and } D H S_{i j} \\ A_{i j} & \text { distance in the y plane between items } i \text { and } j \text { if } i \text { is above } j \\ B_{i j} & \text { distance in the y plane between items } i \text { and } j, \text { if } i \text { is below } j \\ C_{i}^{A L} & \text { direct asset loss of item } i \\ C_{i}^{D E C} & \text { total cost attributed to domino escalation on unit } i \\ C r_{i j} & \text { credibility that the failure of unit } i \text { affects } j \\ d_{i} & \text { breadth of item } i \\ d_{i j h e}^{H} & \text { effective distance between items } i \text { and } j \text { for primary event } e \\ d r_{i j e} & \text { under hazard scenario } h \\ & \text { distance of item } j \text { from the flame envelope/explosion source } \\ D_{i j} & \text { produced by } i \text { due to primary event } e \in\{P F, J F, B W\} \\ D n_{i j} & \text { vertical distance between connection points of items } i \text { and } j, \\ D H I_{i} & \text { if } i \text { is below } j \\ D H S_{i j} & \text { vertical separation distance between items } i \text { and } j, \text { if } i \text { is on a } \\ & \text { lower floor than } j \\ \text { Domino Hazard Index for item } i \\ \text { maximum Domino Hazard Score of item } i \text { with respect to } j \text { for }\end{array}$


$D H S_{i j e}^{E} \quad$ Domino Hazard Score of item $i$ for event $e$ with reference to $j$ $D H S_{i j h e}^{H} \quad$ Domino Hazard Score for an event $e$ under hazard scenario $h$ emanating from item $i$ to $j$

$F A \quad$ base land area

$l_{i} \quad$ length of item $i$

$L_{i j} \quad$ distance in the $\mathrm{x}$ plane between items $i$ and $j$, if $i$ is to the left of $j$

$N Q_{s} \quad$ linearisation variable expressing the product of $N F$ and $Q_{s}$

$R_{i j} \quad$ distance in the $\mathrm{x}$ plane between items $i$ and $j$, if $i$ is to the right of $j$

$T D_{i j} \quad$ total rectilinear distance between items $i$ and $j$ for connection considerations

$T D_{i j}^{S} \quad$ total rectilinear distance between items $i$ and $j$ for safety considerations

$U_{i j} \quad$ vertical distance between connection points of items $i$ and $j$, if $i$ is above $j$

$U p_{i j} \quad$ vertical separation distance between items $i$ and $j$, if $i$ is on a higher floor than $j$

$V D_{i j} \quad$ total vertical distance between items $i$ and $j$

$x_{i}, y_{i} \quad \mathrm{x}, \mathrm{y}$ coordinates of the geometrical centre of item $i$

$X^{\max }, Y^{\max }$ dimensions of base land area

$X Y_{i j}^{\max }$ maximum value between the horizontal separation distance between items $i$ and $j$ in the $\mathrm{x}$ - and $\mathrm{y}$ - planes

$X D_{i j}, Y D_{i j}$ total horizontal distance between the boundaries of items $i$ and $j$ in the $\mathrm{x}, \mathrm{y}$ directions respectively

$Z_{i j}^{+}, Z_{i j}^{-} \quad$ variables to evaluate the difference between $X Y_{i j}^{\max }$ and $V D_{i j}$ if $X Y_{i j}^{\max }>V D_{i j}$ and $X Y_{i j}^{\max }<V D_{i j}$ respectively 


\section{Appendix A. MILP model for multi-floor process plant layout without safety considerations}

The MILP model for multi-floor process plant layout without safety considerations - model OPTL - (Ejeh et al., 2019b) is described below.

\section{A.1. Floor constraints}

Every non-multi-floor equipment $i$ available should be assigned to one floor:

$$
\sum_{k} V_{i k}=1 \quad \forall i \notin I^{T}
$$

Tall/multi-floor equipment items $\left(i \in I^{T}\right)$ are allowed to extend through consecutive floors even if such additional floors are not made available:

$$
\sum_{k} V_{i k}=M_{i}-\omega_{i} \quad \forall i \in I^{T}
$$

where:

$$
\omega_{i} \geq \sum_{k} k \cdot S_{i k}^{S}+M_{i}-|K|-1 \quad \forall i \in I^{T}
$$

A variable, $N_{i j}$, is introduced to determine if equipment $i$ and $j$ occupy the same floor:

$$
N_{i j} \geq V_{i k}+V_{j k}-1 \quad \forall i, j>i, k
$$

The variable $N_{i j}$ takes the value of 1 if and only if items $i$ and $j$ are on any same floor.

Furthermore, a floor must exist if an equipment starts on it:

$$
S_{i k}^{S} \leq W_{k} \quad \forall i, k
$$

or the floor above it is also occupied:

$$
W_{k} \leq W_{k-1} \quad \forall k>1
$$

The minimum number of floors required is then given by:

$$
N F \geq \sum_{k} W_{k}
$$

\section{A.2. Multi-floor equipment constraints}

Equipment items requiring more than one floor are described with the constraints below:

$$
V_{i k}=\sum_{\theta=1}^{M_{i}} \delta_{i \theta} \cdot S_{i, k-\theta+1}^{S} \quad \forall i, k
$$


where $V_{i k}$ is a binary variable which determines if an equipment $i$ is assigned to floor $k$ and $\delta_{i \theta}=1$ for all $\theta \leq M_{i}$. $S_{i k}^{S}$ is a binary variable which determines if an equipment item $i$ starts at floor $k$. This should only occur on one floor:

$$
\sum_{k} S_{i k}^{S}=1 \quad \forall i
$$

These constraints also ensure tall units occupy consecutive floors.

\section{A.3. Equipment orientation constraints}

A $90^{\circ}$ rotation of equipment orientation is allowed in the $\mathrm{x}-\mathrm{y}$ plane:

$$
\begin{array}{cc}
l_{i}=\alpha_{i} O_{i}+\beta_{i}\left(1-O_{i}\right) & \forall i \\
d_{i}=\alpha_{i}+\beta_{i}-l_{i} & \forall i
\end{array}
$$

\section{A.4. Non-overlapping constraints}

To prevent equipment overlap on the same floor, constraints (A.12) - (A.15) are introduced:

$$
\begin{array}{lr}
x_{i}-x_{j}+B M \cdot\left(1-N_{i j}+E 1_{i j}+E 2_{i j}\right) \geq \frac{l_{i}+l_{j}}{2}+D e_{i j}^{\text {min }} & \forall i, j>i \\
x_{j}-x_{i}+B M \cdot\left(2-N_{i j}-E 1_{i j}+E 2_{i j}\right) \geq \frac{l_{i}+l_{j}}{2}+D e_{i j}^{\text {min }} & \forall i, j>i \\
y_{i}-y_{j}+B M \cdot\left(2-N_{i j}+E 1_{i j}-E 2_{i j}\right) \geq \frac{d_{i}+d_{j}}{2}+D e_{i j}^{\text {min }} & \forall i, j>i \\
y_{j}-y_{i}+B M \cdot\left(3-N_{i j}-E 1_{i j}-E 2_{i j}\right) \geq \frac{d_{i}+d_{j}}{2}+D e_{i j}^{\min } & \forall i, j>i
\end{array}
$$

To ensure a globally optimal solution, the value of $B M$ should be such that:

$$
B M \geq \max _{s}\left(\bar{X}_{s}, \bar{Y}_{s}\right)+\max _{i j}\left(D e_{i j}^{\min }\right)
$$

\section{A.5. Distance constraints}

Distance constraints are described by equations (A.17) - (A.19), and determine the distances in the $\mathrm{x}$ and $\mathrm{y}$ planes respectively.

$$
\begin{array}{cc}
R_{i j}-L_{i j}=x_{i}-x_{j} & \forall(i, j) \in \Gamma^{C} \\
A_{i j}-B_{i j}=y_{i}-y_{j} & \forall(i, j) \in \Gamma^{C} \\
T D_{i j}^{C}=R_{i j}+L_{i j}+A_{i j}+B_{i j}+U_{i j}+D_{i j} & \forall(i, j) \in \Gamma^{C}
\end{array}
$$


Provision is made for connection between equipment $i$ and $j$ at design-specified heights of either equipment items as follows:

$$
U_{i j}-D_{i j}=F H \sum_{k}(k-1)\left(S_{i k}^{S}-S_{j k}^{S}\right)+O P_{i j}-I P_{i j} \quad \forall(i, j) \in \Gamma^{C}
$$

where $O P_{i j}$ represents the vertical distance from the base of equipment item $i$ to its output point, and $I P_{i j}$ represents the vertical distance from the base of equipment $j$ to its input point for connection $i-j$.

\section{A.6. Layout design constraints}

Layout design constraints, (A.21) - (A.24), ensure that equipment are placed within the boundaries of a floor:

$$
\begin{array}{ll}
x_{i}+\frac{l_{i}}{2} \leq X^{\max } & \forall i \\
y_{i}+\frac{d_{i}}{2} \leq Y^{\max } & \forall i
\end{array}
$$

with a lower bound:

$$
\begin{array}{rlrl}
x_{i} & \geq \frac{l_{i}}{2} & \forall i \\
y_{i} \geq \frac{d_{i}}{2} & \forall i
\end{array}
$$

\section{A.7. Area Constraints}

In order to avoid bilinear terms in calculating the floor area, $F A$, equations (A.25) - (A.29) are introduced. The area of each floor is determined from a set $S$ of predefined rectangular area sizes, $A R_{s}$, with dimensions $\left(\bar{X}_{s}, \bar{Y}_{s}\right)$.

$$
\begin{gathered}
F A=\sum_{s} A R_{s} Q_{s} \\
\sum_{s} Q_{s}=1
\end{gathered}
$$

The floor length and breadth is selected from the chosen rectangular area size dimensions:

$$
\begin{aligned}
X^{\max } & =\sum_{s} \bar{X}_{s} Q_{s} \\
Y^{\max } & =\sum_{s} \bar{Y}_{s} Q_{s}
\end{aligned}
$$

Also, a new term $N Q_{s}$ is introduced in order to linearise the cost term associated with the number of floors:

$$
\begin{gathered}
N F=\sum_{s} N Q_{s} \\
N Q_{s} \leq|K| \cdot Q_{s} \quad \forall s
\end{gathered}
$$




\section{A.8. Symmetry breaking constraints}

To reduce the occurrence of symmetric solutions, the following constraints are introduced:

$$
\begin{gathered}
x_{i}+y_{i}-x_{j}-y_{j} \geq \sigma \cdot N_{i j} \quad \forall(i, j)=\underset{i \in I^{T}, j \in I^{T}}{\arg \max } C_{i j}^{c} \\
E 1_{i j}=0 \quad \forall(i, j)=\underset{i \in I^{T}, j \in I^{T}}{\arg \max } C_{i j}^{c}
\end{gathered}
$$

\section{A.9. Integer cut}

The following integer cuts were applied to the model to reduce the solution space by eliminating unrealistic overlap considerations:

$$
\begin{array}{rlr}
\frac{E 1_{i n}+E 2_{i n}}{2} & \geq E 1_{i j}+E 2_{i j}+E 1_{j n}+E 2_{j n}-3 \quad \forall i<j<n \\
N_{i j} & \geq E 1_{i j} \quad \forall i, j>i \\
N_{i j} & \geq E 2_{i j} \quad \forall i, j>i
\end{array}
$$

\section{A.10. Objective function}

The objective function minimises the total connection cost, pumping cost, land area cost, floor construction cost and floor-area dependent cost:

$$
\begin{array}{r}
\min \sum_{i} \sum_{(i, j) \in \Gamma^{C}}\left[C_{i j}^{C} T D_{i j}^{C}+C_{i j}^{V} D_{i j}+C_{i j}^{H}\left(R_{i j}+L_{i j}+A_{i j}+B_{i j}\right)\right] \\
+F C 1 \cdot N F+F C 2 \sum_{s} A R_{s} \cdot N Q_{s}+L C \cdot F A
\end{array}
$$

subject to equations (A.1) - (A.35). 


\section{Appendix B. Supplementary information on proposed MILP model}

B.1. Pool \&5 Jet fire: Parameters for piecewise linear approximation

Table B.1: Piecewise parameters for evaluation of DHS for Pool/Jet fire scenarios

\begin{tabular}{|c|c|c|c|c|c|c|c|c|c|}
\hline & \multirow[b]{3}{*}{$\hat{q}$} & \multicolumn{4}{|c|}{ Atmospheric equipment } & \multicolumn{4}{|c|}{ Pressurized equipment } \\
\hline & & \multicolumn{2}{|c|}{ Unprotected } & \multicolumn{2}{|c|}{ FI } & \multicolumn{2}{|c|}{ Unprotected } & \multicolumn{2}{|c|}{ FI } \\
\hline & & $\kappa_{i h e \hat{g}}^{A}$ & $\lambda_{i h e \hat{q}}^{A}$ & $\kappa_{i h e \hat{q}}^{A}$ & $\lambda_{i h e \hat{g}}^{A}$ & $\kappa_{i h e \hat{q}}^{P}$ & $\lambda_{i h e \hat{q}}^{P}$ & $\kappa_{i h e \hat{q}}^{P}$ & $\lambda_{i h e \hat{q}}^{P}$ \\
\hline \multirow{4}{*}{$\begin{array}{l}\text { Pool fire } \\
\text { scenario }\end{array}$} & 1 & 0 & 0 & 0 & 0 & 0 & 0 & 0 & 0 \\
\hline & 2 & 0.5 & 4.5 & 7.6 & 4.5 & 1.4 & 4.5 & 7.8 & 4.5 \\
\hline & 3 & 2.5 & 35.5 & 0.6 & 35.5 & 3 & 11.7 & 0.8 & 11.7 \\
\hline & 4 & 7 & 10 & 1.8 & 10 & 5.6 & 2.8 & 1.4 & 2.8 \\
\hline \multirow{4}{*}{$\begin{array}{l}\text { Jet fire } \\
\text { scenario }\end{array}$} & 1 & 0 & 0 & 0 & 0 & 0 & 0 & 0 & 0 \\
\hline & 2 & 1 & 5 & 7.6 & 5 & 3 & 5 & 8.2 & 5 \\
\hline & 3 & 2.2 & 40 & 0 & 40 & 2.2 & 10 & 0.6 & 10 \\
\hline & 4 & 6.8 & 5 & 1.8 & 5 & 4.8 & 4 & 1.2 & 4 \\
\hline
\end{tabular}

Source: de Lira-Flores et al. (2014)

\section{B.2. Additional constraints for safety considerations}

The effective distances, $d_{i j h e}^{H}$, for each hazard scenario $h$ and event $e$ matching the disjunctions represented by equations (2) - (5), (7), and (11), are formulated as follows.

\section{B.2.1. Flash fire}

For flash fire events, the distance between two equipment items for an effective hazard scenario $h, d_{i j h, F F}^{H}$, is evaluated as follows. The separation distance, $T D_{i j}^{S}$, is the sum of these distances for scenarios associated with flash fires $\left(h \in H_{F F}\right.$, where $\left.H_{F F}=\{H Z 1, S F\}\right)$ :

$$
T D_{i j}^{S}=\sum_{h \in H_{F F}} d_{i j h, F F}^{H} \quad \forall(i, j) \in \Gamma^{H}, j \in I^{V}
$$

The distances for each scenario must correspond to the permitted range as described in equation (2):

$$
\begin{gathered}
d_{i j, H Z 1, F F}^{H} \leq d_{F F, i}^{F} \cdot B_{i j, H Z 1, F F}^{L} \quad \forall(i, j) \in \Gamma^{H}, j \in I^{V} \\
d_{i j, S F, F F}^{H} \geq\left(d_{F F, i}^{F}+\digamma\right) \cdot B_{i j, S F, F F}^{L} \quad \forall(i, j) \in \Gamma^{H}, j \in I^{V} \\
d_{i j, S F, F F}^{H} \leq B M \cdot B_{i j, S F, F F}^{L} \quad \forall(i, j) \in \Gamma^{H}, j \in I^{V}
\end{gathered}
$$




\section{B.2.2. Fireball}

For fireball events, the total separation distance between units must equal the sum of the effective distances for each potential hazard scenario:

$$
T D_{i j}^{S}=\sum_{h \in H_{F B}} d_{i j h, F B}^{H} \quad \forall(i, j) \in \Gamma^{H}, j \in I^{A}
$$

where $H_{F B}=\{H Z 1, F I, S F\}$. If the separation distance falls within the allowable limits of a hazard condition it is assigned according:

$$
\begin{gathered}
d_{i j, H Z 1, F B}^{H} \leq d_{F B, i}^{F} \cdot B_{i j, H Z 1, F B}^{L} \quad \forall(i, j) \in \Gamma^{H}, j \in I^{A} \\
d_{i j, F I, F B}^{H} \leq d_{F B, i}^{F} \cdot B_{i j, F I, F B}^{L} \quad \forall(i, j) \in \Gamma^{H}, j \in I^{A} \\
d_{i j, S F, F B}^{H} \geq\left(d_{F B, i}^{F}+\digamma\right) \cdot B_{i j, S F, F B}^{L} \quad \forall(i, j) \in \Gamma^{H}, j \in I^{A} \\
d_{i j, S F, F B}^{H} \leq B M \cdot B_{i j, S F, F B}^{L} \quad \forall(i, j) \in \Gamma^{H}, j \in I^{A}
\end{gathered}
$$

where $\digamma$ is a very small number included to force a strict inequality, and $B M$ is a large number.

\section{B.2.3. Pool}

For a pool fire event, the separation distance, $d r_{i j, P F}$, between an item $j$ and the flame envelope produced from $i$ is given by:

$$
d r_{i j, P F}=T D_{i j}^{S}-d_{P F, i}^{F} \quad \forall i \in I^{V},(i, j) \in \Gamma^{H}
$$

This distance is equal to the sum of effective distances for each potential hazard scenario in a pool fire event:

$$
d r_{i j, P F}=\sum_{h \in H_{P F}} d_{i j h, P F}^{H} \quad \forall i \in I^{V},(i, j) \in \Gamma^{H}
$$

where $H_{P F}=\{H Z 1, H Z 2, F I, F W, S F\}$. For HZ1 scenario to occur, the secondary item $j$ has be within direct reach of the flame envelope:

$$
d_{i j, H Z 1, P F}^{H} \leq 0 \quad \forall i \in I^{V},(i, j) \in \Gamma^{H}
$$

HZ2 and FI scenarios corresponds to item $j$ being within reach of the effect of heat radiation $\left(0<d r_{i j e} \leq d_{e j}^{S}\right)$ and having no device protection or a fire insulation installed respectively. Using a piecewise linearisation approach, for atmospheric secondary item $j$ :

$$
d_{i j h, P F}^{H}=\sum_{\hat{q}} \lambda_{i h e \hat{q}}^{A} \cdot \mu_{i j h, P F, \hat{q}} \quad \forall i \in I^{V},(i, j) \in \Gamma^{H}, j \in I^{A}, h \in\{H Z 2, F I\}
$$

For pressurized secondary items:

$$
d_{i j h, P F}^{H}=\sum_{\hat{q}} \lambda_{i h e \hat{q}}^{P} \cdot \mu_{i j h, P F, \hat{q}} \quad \forall i \in I^{V},(i, j) \in \Gamma^{H}, j \in I^{P}, h \in\{H Z 2, F I\}
$$


The piecewise linearisation variable $\mu_{i j h e \hat{q}}$ is evaluated as follows (de Lira-Flores et al., 2014):

$$
\begin{aligned}
& \mu_{i j h, P F, 1} \leq 1 \forall i \in I^{V},(i, j) \in \Gamma^{H}, h \in\{H Z 2, F I\} \\
& \mu_{i j h, P F, 4} \geq 0 \quad \forall i \in I^{V},(i, j) \in \Gamma^{H}, h \in\{H Z 2, F I\} \\
& \mu_{i j h, P F, \hat{q}+1} \leq B_{i j h, P F, \hat{q}}^{F} \quad \forall i \in I^{V},(i, j) \in \Gamma^{H}, h \in\{H Z 2, F I\} \\
& B_{i j h, P F, \hat{q}}^{F} \leq \mu_{i j h, P F, 1} \quad \forall i \in I^{V},(i, j) \in \Gamma^{H}, h \in\{H Z 2, F I\} \\
& B_{i j h, P F, \hat{q}}^{F} \leq B_{i j h, P F}^{L} \quad \forall i \in I^{V},(i, j) \in \Gamma^{H}, h \in\{H Z 2, F I\} \\
& \digamma \cdot B_{i j h P F}^{L} \leq d_{i j h, P F}^{H} \quad \forall i \in I^{V},(i, j) \in \Gamma^{H}, h \in\{H Z 2, F I\} \\
& d_{i j h, P F}^{H} \leq d_{P F, j}^{S} B_{i j h, P F}^{L} \quad \forall i \in I^{V},(i, j) \in \Gamma^{H}, h \in\{H Z 2, F I\}
\end{aligned}
$$

FW scenarios correspond to the same conditions as the HZ2 scenario with the addition of a firewall being installed for item $j$.

$$
d_{i j, F W, P F}^{H} \leq d_{P F, i}^{S} B_{i j, F W, P F}^{L} \quad \forall i \in I^{V},(i, j) \in \Gamma^{H}
$$

Finally, if the secondary item is out of reach of the effects of radiation and direct flame impingement, the distance is obtained as follows:

$$
\begin{aligned}
\left(d_{P F, j}^{S}+\digamma\right) \cdot B_{i j, S F, P F}^{L} \leq d_{i j, S F, P F}^{H} & \forall i \in I^{V},(i, j) \in \Gamma^{H} \\
d_{i j, S F, P F}^{H} \leq B M \cdot B_{i j, S F, P F}^{L} & \forall i \in I^{V},(i, j) \in \Gamma^{H}
\end{aligned}
$$

Additional constraints ((B.25) - (B.26)) are included to ensure that separation distance values are assigned to the correct hazard scenario:

$$
\begin{array}{r}
d_{i j h, P F}^{H} \leq d_{P F, i}^{S}\left(B_{i j, H Z 2, P F}^{L}+B_{i j, F I, P F}^{L}+B_{i j, F W, P F}^{L}\right)+B M \cdot B_{i j, S F, P F}^{L} \\
\forall i \in I^{V},(i, j) \in \Gamma^{H}, h \in H_{P F} \\
d_{i j h, P F}^{H} \geq-d_{P F, i}^{F} \cdot\left(B_{i j, H Z 1, P F}^{L}+B_{i j, F W, P F}^{L}\right)+\left(d_{P F, i}^{S}+\digamma\right) \cdot B_{i j, S F, P F}^{L} \\
\forall i \in I^{V},(i, j) \in \Gamma^{H}, h \in H_{P F}
\end{array}
$$

\section{B.2.4. Blast wave}

The effective distance for each hazard scenario for BW events are evaluated as follows. The distance from the explosion source $d r_{i j, B W}$ is given by:

$$
d r_{i j, B W}=T D_{i j}^{s}-d_{B W, i}^{F} \quad \forall(i, j) \in \Gamma^{H}
$$

The sum of the effective distances for each hazard scenario, $d_{i j h, B W}^{H}$, must be equal to the distance from the explosion source both for atmospheric and pressurized secondary items.

$$
d r_{i j, B W}=\sum_{h \in H_{B W}} d_{i j h, B W}^{H}+\left.d_{i j, H Z 2, B W}^{H}\right|_{j \in I^{A}} \quad \forall(i, j) \in \Gamma^{H}
$$


where $H_{B W}=\{H Z 1, B W l, S F\}$. For HZ1 scenario, the secondary item is within the upper distance limit for pressurized secondary items, and the lower distance limit for atmospheric secondary items.

$$
\begin{gathered}
d_{i j, H Z 1, B W}^{H} \geq 0 \quad \forall(i, j) \in \Gamma^{H} \\
d_{i j, H Z 1, B W}^{H} \leq\left(\left.\left(P_{i}^{l o}-\digamma\right)\right|_{j \in I^{A}}+\left.P_{i}^{u p}\right|_{j \in I^{P}}\right) \cdot B_{i j, H Z 1, B W}^{L} \quad \forall(i, j) \in \Gamma^{H}
\end{gathered}
$$

HZ2 scenario only occurs for atmospheric secondary equipment items if the distance from the explosion source is within the upper and lower distance limits $\left(P_{i}^{u p}, P_{i}^{l o}\right)$ :

$$
\begin{gathered}
P_{i}^{l o} \cdot B_{i j, H Z 2, B W}^{L} \leq d_{i j, H Z 2, B W}^{H} \quad \forall(i, j) \in \Gamma^{H}, j \in I^{A} \\
d_{i j, H Z 2, B W}^{H} \leq P_{i}^{u p} \cdot B_{i j, H Z 2, B W}^{L} \quad \forall(i, j) \in \Gamma^{H}, j \in I^{A}
\end{gathered}
$$

For BWl scenarios, the separation distance from the explosion source is within the upper distance limit for overpressure:

$$
\begin{gathered}
d_{i j, B W l, B W}^{H} \geq 0 \quad \forall(i, j) \in \Gamma^{H} \\
d_{i j, B W l, B W}^{H} \leq P_{i}^{u p} \cdot B_{i j, B W l, B W}^{L} \quad \forall(i, j) \in \Gamma^{H}
\end{gathered}
$$

Finally, a secondary item is considered safe from overpressure if its distance to the point of explosion is greater than the upper overpressure distance limit:

$$
\begin{gathered}
\left(P_{i}^{u p}+\digamma\right) B_{i j, S F, B W}^{L} \leq d_{i j, S F, B W}^{H} \quad \forall(i, j) \in \Gamma^{H} \\
d_{i j, S F, B W}^{H} \leq B M \cdot B_{i j, S F, B W}^{L} \quad \forall(i, j) \in \Gamma^{H}
\end{gathered}
$$

where $B M$ is a big number and $\digamma$ is a very small number.

\section{B.3. Constraints for Jet fires}

The DHS for each potential hazard scenario $h$ in a jet fire event, $D H S_{i j h, J F}^{H}$, is evaluated using equations (B.37) - (B.60) similar to pool fires:

$$
\begin{aligned}
D H S_{i j, J F}^{e} & =\sum_{h \in H_{J F}} D H S_{i j h, J F}^{H} \quad \forall i \in I^{V},(i, j) \in \Gamma^{H} \\
d r_{i j, J F} & =T D_{i j}^{S}-d_{J F, i}^{F} \quad \forall i \in I^{V},(i, j) \in \Gamma^{H} \\
d r_{i j, J F} & =\sum_{h \in H_{J F}} d_{i j h, J F}^{H} \quad \forall i \in I^{V},(i, j) \in \Gamma^{H}
\end{aligned}
$$

where $H_{J F}=\{H Z 1, H Z 2, F I, F W, S F\}$.

$$
\begin{gathered}
D H S_{i j, H Z 1, J F}^{H}=10 \cdot B_{i j, H Z 1, J F}^{L} \quad \forall i \in I^{V},(i, j) \in \Gamma^{H} \\
d_{i j, H Z 1, J F}^{H} \leq 0 \quad \forall i \in I^{V},(i, j) \in \Gamma^{H}
\end{gathered}
$$




$$
\begin{gathered}
D H S_{i j, F W, J F}^{H}=B_{i j, F W, J F}^{L} \quad \forall i \in I^{V},(i, j) \in \Gamma^{H} \\
d_{i j, F W, J F}^{H} \leq d_{J F, i}^{S} B_{i j, F W, J F}^{L} \quad \forall i \in I^{V},(i, j) \in \Gamma^{H} \\
D H S_{i j h J F}^{H}=10 \cdot B_{i j h J F}^{L}-\sum_{\hat{q}} \kappa_{i h, J F, \hat{q}}^{A} \cdot \mu_{i j h, J F, \hat{q}} \\
\forall i \in I^{V},(i, j) \in \Gamma^{H}, j \in I^{A}, h \in\{H Z 2, F I\} \\
d_{i j h, J F}^{H}=\sum_{\hat{q}} \lambda_{i h e \hat{q}}^{A} \cdot \mu_{i j h, J F, \hat{q}} \quad \forall i \in I^{V},(i, j) \in \Gamma^{H}, j \in I^{A}, h \in\{H Z 2, F I\} \\
D H S_{i j h, J F}^{H}=10 \cdot B_{i j h, J F}^{L}-\sum_{\hat{q}} \kappa_{i h, J F, \hat{q}}^{P} \cdot \mu_{i j h, J F, \hat{q}} \\
\forall i \in I^{V},(i, j) \in \Gamma^{H}, j \in I^{P}, h \in\{H Z 2, F I\} \\
d_{i j h, J F}^{H}=\sum_{\hat{q}} \lambda_{i h e \hat{q}}^{P} \cdot \mu_{i j h, J F, \hat{q}} \quad \forall i \in I^{V},(i, j) \in \Gamma^{H}, j \in I^{P}, h \in\{H Z 2, F I\}
\end{gathered}
$$

$$
\begin{aligned}
& \mu_{i j h, J F, 1} \leq 1 \quad \forall i \in I^{V},(i, j) \in \Gamma^{H}, h \in\{H Z 2, F I\} \\
& \mu_{i j h, J F, 4} \geq 0 \quad \forall i \in I^{V},(i, j) \in \Gamma^{H}, h \in\{H Z 2, F I\} \\
& \mu_{i j h, J F, \hat{q}+1} \leq B_{i j h, J F, \hat{q}}^{F} \quad \forall i \in I^{V},(i, j) \in \Gamma^{H}, h \in\{H Z 2, F I\} \\
& B_{i j h, J F, \hat{q}}^{F} \leq \mu_{i j h, J F, 1} \quad \forall i \in I^{V},(i, j) \in \Gamma^{H}, h \in\{H Z 2, F I\} \\
& B_{i j h, J F, \hat{q}}^{F} \leq B_{i j h, J F}^{L} \quad \forall i \in I^{V},(i, j) \in \Gamma^{H}, h \in\{H Z 2, F I\} \\
& \digamma \cdot B_{i j h J F}^{L} \leq d_{i j h, J F}^{H} \quad \forall i \in I^{V},(i, j) \in \Gamma^{H}, h \in\{H Z 2, F I\} \\
& d_{i j h, J F}^{H} \leq d_{J F, j}^{S} B_{i j h, J F}^{L} \quad \forall i \in I^{V},(i, j) \in \Gamma^{H}, h \in\{H Z 2, F I\} \\
& \left(d_{J F, j}^{S}+\digamma\right) \cdot B_{i j, S F, J F}^{L} \leq d_{i j, S F, J F}^{H} \quad \forall i \in I^{V},(i, j) \in \Gamma^{H} \\
& d_{i j, S F, J F}^{H} \leq B M \cdot B_{i j, S F, J F}^{L} \quad \forall i \in I^{V},(i, j) \in \Gamma^{H} \\
& d_{i j h, J F}^{H} \leq d_{J F, i}^{S}\left(B_{i j, H Z 2, J F}^{L}+B_{i j, F I, J F}^{L}+B_{i j, F W, J F}^{L}\right)+B M \cdot B_{i j, S F, J F}^{L} \\
& \forall i \in I^{V},(i, j) \in \Gamma^{H}, h \in H_{J F} \\
& d_{i j h, J F}^{H} \geq-d_{J F, i}^{F} \cdot\left(B_{i j, H Z 1, J F}^{L}+B_{i j, F W, J F}^{L}\right)+\left(d_{J F, i}^{S}+\digamma\right) \cdot B_{i j, S F, J F}^{L} \\
& \forall i \in I^{V},(i, j) \in \Gamma^{H}, h \in H_{J F} \\
& \sum_{h \in H_{J F}} B_{i j h, J F}^{L}=1 \quad \forall i \in I^{V},(i, j) \in \Gamma^{H} \\
& B_{i j, F W, J F}^{L}+B_{i j, F I, J F}^{L}+B_{i j, S F, J F}^{L} \geq 1-N_{i j} \quad \forall i \in I^{V},(i, j) \in \Gamma^{H}
\end{aligned}
$$




\section{Appendix C. Data for case study}

Table C.1 shows the dimensions of equipment items and Table C.3 shows the connection cost, connection heights, horizontal and vertical pumping costs, as well as other required data for the acrylic acid (AA) plant.

Table C.1: Equipment dimensions for the AA plant

\begin{tabular}{clccc}
\hline $\begin{array}{c}\text { Equipment } \\
\text { item }\end{array}$ & Description & $\alpha_{i}(\mathrm{~m})$ & $\beta_{i}(\mathrm{~m})$ & $\gamma_{i}(\mathrm{~m})$ \\
\hline 1 & Compressor & 2.0 & 2.0 & 2.0 \\
2 & Feed mixer & 2.5 & 2.5 & 3.5 \\
3 & Reactor & 2.8 & 2.8 & 5.2 \\
4 & Quench & 3.3 & 3.3 & 6.4 \\
5 & Absorber & 2.5 & 2.5 & 7.0 \\
6 & Splitter & 3.1 & 3.1 & 3.1 \\
7 & Acid extractor & 2.5 & 2.5 & 5.5 \\
8 & Distillation column 1 & 3.0 & 3.0 & 7.2 \\
9 & Solvent mixer & 1.8 & 1.8 & 1.8 \\
10 & Distillation column 2 & 2.5 & 2.5 & 5.8 \\
11 & Distillation column 3 & 2.2 & 2.2 & 4.7 \\
\hline
\end{tabular}

Table C.2: Equipment item parameters for safety considerations

(a) Part 1

\begin{tabular}{clccccc}
\hline$i$ & Description & $D I_{i}$ & $C_{i}^{P}(\mathrm{rmu})$ & $C_{i, F I}(\mathrm{rmu})$ & $C_{i, F W}(\mathrm{rmu})$ & $C_{i, B W l}(\mathrm{rmu})$ \\
\hline 1 & Compressor & 7 & 6,000 & 563 & 8,000 & 8,000 \\
2 & Feed mixer & 29 & 41,735 & 2,661 & 20,000 & 58,000 \\
3 & Reactor & 47 & 88,906 & 2,925 & 20,000 & 61,429 \\
4 & Quench & 25 & 45,836 & 2,787 & 20,000 & 53,636 \\
5 & Absorber & 30 & 62,335 & 2,212 & 20,000 & 48,400 \\
6 & Splitter & 21 & 23,365 & 1,640 & 15,000 & 30,645 \\
7 & Acid Extractor & 30 & 67,876 & 2,506 & 20,000 & 54,800 \\
8 & Distillation 1 & 22 & 94,909 & 2,117 & 20,000 & 42,000 \\
9 & Solvent mixer & 20 & 33,417 & 2,273 & 20,000 & 54,444 \\
10 & Distillation 2 & 21 & 48,898 & 2,212 & 20,000 & 48,400 \\
11 & Distillation 3 & 27 & 59,236 & 2,351 & 20,000 & 53,636 \\
\hline
\end{tabular}


(b) Part 2

\begin{tabular}{clcc}
\hline$i$ & Description & $P_{i}^{l o}(\mathrm{~m})$ & $P_{i}^{u p}(\mathrm{~m})$ \\
\hline 1 & Compressor & - & - \\
2 & Feed mixer & 8.8 & 13.6 \\
3 & Reactor & 19.5 & 30.0 \\
4 & Quench & 14.8 & 22.9 \\
5 & Absorber & 14.5 & 22.7 \\
6 & Splitter & 5.6 & 8.4 \\
7 & Acid Extractor & - & - \\
8 & Distillation 1 & 22.0 & 16.0 \\
9 & Solvent mixer & - & - \\
10 & Distillation 2 & 13.0 & 9.0 \\
11 & Distillation 3 & 22.0 & 16.0 \\
\hline
\end{tabular}

Table C.3: Parameters for the AA plant

(a) Connection and pumping costs, and connection heights

\begin{tabular}{cccccc}
\hline Connection & $C_{i j}^{C}(\mathrm{rmu} / \mathrm{m})$ & $C_{i j}^{H}(\mathrm{rmu} / \mathrm{m})$ & $C_{i j}^{V}(\mathrm{rmu} / \mathrm{m})$ & $O P_{i j}(\mathrm{~m})$ & $I P_{i j}(\mathrm{~m})$ \\
\hline 1.2 & 166.13 & 863.876 & $8,638.76$ & 0.00 & 3.50 \\
2.3 & 166.13 & 863.876 & $8,638.76$ & 0.00 & 2.60 \\
3.4 & 166.13 & 863.876 & $8,638.76$ & 0.00 & 3.20 \\
4.5 & 166.13 & 863.876 & $8,638.76$ & 0.00 & 3.50 \\
5.6 & 166.13 & 863.876 & $8,638.76$ & 7.00 & 1.55 \\
5.7 & 166.13 & 863.876 & $8,638.76$ & 0.00 & 2.75 \\
6.2 & 166.13 & 863.876 & $8,638.76$ & 1.55 & 3.50 \\
7.8 & 166.13 & 863.876 & $8,638.76$ & 5.50 & 3.60 \\
7.11 & 166.13 & 863.876 & $8,638.76$ & 0.00 & 2.35 \\
8.9 & 166.13 & 863.876 & $8,638.76$ & 7.20 & 0.90 \\
8.10 & 166.13 & 863.876 & $8,638.76$ & 0.00 & 2.90 \\
11.9 & 166.13 & 863.876 & $8,638.76$ & 4.70 & 0.90 \\
\hline
\end{tabular}

(b) Other Parameters

\begin{tabular}{lr}
\hline Parameters & Value \\
\hline$|K|$ & 4 \\
$F C 1(\mathrm{rmu})$ & 3,330 \\
$F C 2\left(\mathrm{rmu} / \mathrm{m}^{2}\right)$ & 1,000 \\
$L C\left(\mathrm{rmu} / \mathrm{m}^{2}\right)$ & 5,000 \\
$F H(\mathrm{~m})$ & 5.0 \\
\hline
\end{tabular}




\section{References}

Ahumada, C. B., Quddus, N., Mannan, M. S., 2018. A method for facility layout optimisation including stochastic risk assessment. Process Saf. Environ. Prot. $117,616-628$.

Almaraz, S. D.-L., Boix, M., Montastruc, L., Azzaro-Pantel, C., Liao, Z., Domenech, S., 2016. Design of a water allocation and energy network for multi-contaminant problems using multi-objective optimization. Process Safety and Environmental Protection 103, 348-364.

American Institute of Chemical Engineers, 1994. Dow's Fire \& Explosion Index Hazard Classification Guide. Vol. 7. John Wiley \& Sons, Inc., Hoboken, NJ, USA.

Barbosa-Póvoa, A. P., Mateus, R., Novais, A. Q., 2002. Optimal 3D layout of industrial facilities. Int. J. Prod. Res. 40, 1669-1698.

Chiandussi, G., Codegone, M., Ferrero, S., Varesio, F. E., 2012. Comparison of multi-objective optimization methodologies for engineering applications. Computers \& Mathematics with Applications 63, 912-942.

Cozzani, V., Gubinelli, G., Salzano, E., 2006. Escalation thresholds in the assessment of domino accidental events. J. Hazard. Mater. 129, 1-21.

D'Ambrosio, C., Lodi, A., Martello, S., 2010. Piecewise linear approximation of functions of two variables in MILP models. Oper. Res. Lett. 38, 39-46.

de Lira-Flores, J., Vázquez-Román, R., López-Molina, A., Mannan, M. S., 2014. A MINLP approach for layout designs based on the domino hazard index. J. Loss Prev. Process Ind. 30, 219-227.

de Lira-Flores, J. A., Gutiérrez-Antonio, C., Vázquez-Román, R., 2018. A MILP approach for optimal storage vessels layout based on the quantitative risk analysis methodology. Process Saf. Environ. Prot. 120, 1-13.

de Lira-Flores, J. A., López-Molina, A., Gutiérrez-Antonio, C., VázquezRomán, R., 2019. Optimal plant layout considering the safety instrumented system design for hazardous equipment. Process Safety and Environmental Protection 124, 97-120.

Díaz-Ovalle, C., Vázquez-Román, R., de Lira-Flores, J., Mannan, M. S., 2013. A model to optimize facility layouts with toxic releases and mitigation systems. Computers \& chemical engineering 56, 218-227.

Ejeh, J. O., Liu, S., Chalchooghi, M. M., Papageorgiou, L. G., 2018a. Optimization-based approach for process plant layout. Ind. Eng. Chem. Res. $57,10482-10490$.

Ejeh, J. O., Liu, S., Papageorgiou, L. G., 2018b. Optimal multi-floor process plant layout with production sections. Chem. Eng. Res. Des. 137, 488-501. 
Ejeh, J. O., Liu, S., Papageorgiou, L. G., 2019a. An MILP model for safe multifloor process plant layout. In: Comput. Aided Chem. Eng. pp. 379-384.

Ejeh, J. O., Liu, S., Papageorgiou, L. G., 2019b. Optimal layout of multi-floor process plants using MILP. Comput. Chem. Eng. 131, 106573.

GAMS Development Corporation, 2018. General Algebraic Modeling System (GAMS) Release 25.0.2.

Georgiadis, M. C., Macchietto, S., 1997. Layout of process plants: A novel approach. Comput. Chem. Eng. 21, S337-S342.

Guirardello, R., Swaney, R. E., 2005. Optimization of process plant layout with pipe routing. Comput. Chem. Eng. 30, 99-114.

Haimes, Y. V., Lasdon, L. S., Wismer, D. A., 1971. On a bicriterion formation of the problems of integrated system identification and system optimization. IEEE Trans. Syst. Man Cybern. SMC-1, 296-297.

Han, K., Cho, S., Yoon, E. S., 2013. Optimal layout of a chemical process plant to minimize the risk to humans. In: Procedia Comput. Sci. Vol. 22. pp. $1146-1155$.

Hosseini-Nasab, H., Fereidouni, S., Fatemi Ghomi, S. M. T., Fakhrzad, M. B., 2018. Classification of facility layout problems: a review study. Int. J. Adv. Manuf. Technol. 94, 957-977.

Hwang, J., Lee, K. Y., 2014. Optimal liquefaction process cycle considering simplicity and efficiency for LNG FPSO at FEED stage. Comput. Chem. Eng. 63, 1-33.

Jafari, M. J., Mohammadi, H., Reniers, G., Pouyakian, M., Nourai, F., Torabi, S. A., Rafiee Miandashti, M., 2018. Exploring inherent process safety indicators and approaches for their estimation: A systematic review. J. Loss Prev. Process Ind. 52, 66-80.

Jung, S., 2016. Facility siting and plant layout optimization for chemical process safety. Korean J. Chem. Eng. 33, 1-7.

Khan, F. I., Amyotte, P. R., 2004. Integrated inherent safety index (I2SI): A tool for inherent safety evaluation. Process Saf. Prog. 23, 136-148.

Khan, F. I., Amyotte, P. R., 2005. I2SI: A comprehensive quantitative tool for inherent safety and cost evaluation. J. Loss Prev. Process Ind. 18, 310-326.

Kidam, K., Hurme, M., 2012. Design as a contributor to chemical process accidents. J. Loss Prev. Process Ind. 25, 655-666.

Latifi, S. E., Mohammadi, E., Khakzad, N., 2017. Process plant layout optimization with uncertainty and considering risk. Comput. Chem. Eng. 106, 224-242. 
Liu, S., Papageorgiou, L. G., 2013. Multiobjective optimisation of production, distribution and capacity planning of global supply chains in the process industry. Omega 41, 369-382.

Medina-Herrera, N., Jiménez-Gutiérrez, A., Grossmann, I. E., 2014. A mathematical programming model for optimal layout considering quantitative risk analysis. Computers \& chemical engineering 68, 165-181.

Papageorgiou, L. G., Rotstein, G. E., 1998. Continuous-domain mathematical models for optimal process plant layout. Ind. Eng. Chem. Res. 37, 3631-3639.

Park, K., Koo, J., Shin, D., Lee, C. J., Yoon, E. S., 2011. Optimal multi-floor plant layout with consideration of safety distance based on mathematical programming and modified consequence analysis. Korean J. Chem. Eng. 28, 1009-1018.

Park, K., Shin, D., Won, W., 2018. Risk based 3-dimensional and multifloor plant layout optimization for liquefied natural gas (LNG) liquefaction process. Korean J. Chem. Eng. 35, 1053-1064.

Patsiatzis, D. I., Knight, G., Papageorgiou, L. G., 2004. An MILP approach to safe process plant layout. Chem. Eng. Res. Des. 82, 579-586.

Patsiatzis, D. I., Papageorgiou, L. G., 2002. Optimal multi-floor process plant layout. Comput. Chem. Eng. 26, 575-583.

Patsiatzis, D. I., Papageorgiou, L. G., 2003. Efficient solution approaches for the multifloor process plant layout problem. Ind. Eng. Chem. Res. 42, 811-824.

Penteado, F. D., Ciric, A. R., 1996. An MINLP approach for safe process plant layout. Ind. Eng. Chem. Res. 35, 1354-1361.

Roy, N., Eljack, F., Jiménez-Gutiérrez, A., Zhang, B., Thiruvenkataswamy, P., El-Halwagi, M., Mannan, M. S., 2016. A review of safety indices for process design. Curr. Opin. Chem. Eng. 14, 42-48.

Tugnoli, A., Khan, F., Amyotte, P., Cozzani, V., 2008. Safety assessment in plant layout design using indexing approach: Implementing inherent safety perspective. Part 1 - Guideword applicability and method description. J. Hazard. Mater. 160, 100-109.

Voll, P., Jennings, M., Hennen, M., Shah, N., Bardow, A., 2015. The optimum is not enough: A near-optimal solution paradigm for energy systems synthesis. Energy 82, 446-456.

Wang, R., Wu, Y., Wang, Y., Feng, X., 2017. An industrial area layout optimization method based on dow's Fire \& Explosion Index Method. In: Chem. Eng. Trans. Vol. 61. pp. $493-498$. 
Wang, R., Wu, Y., Wang, Y., Feng, X., Liu, M., 2019. An layout optimization method for industrial facilities based on domino hazard index. In: Computer Aided Chemical Engineering. Vol. 47. Elsevier, pp. 89-94.

Zhang, D., Evangelisti, S., Lettieri, P., Papageorgiou, L. G., 2016. Economic and environmental scheduling of smart homes with microgrid: Der operation and electrical tasks. Energy conversion and management 110, 113-124. 